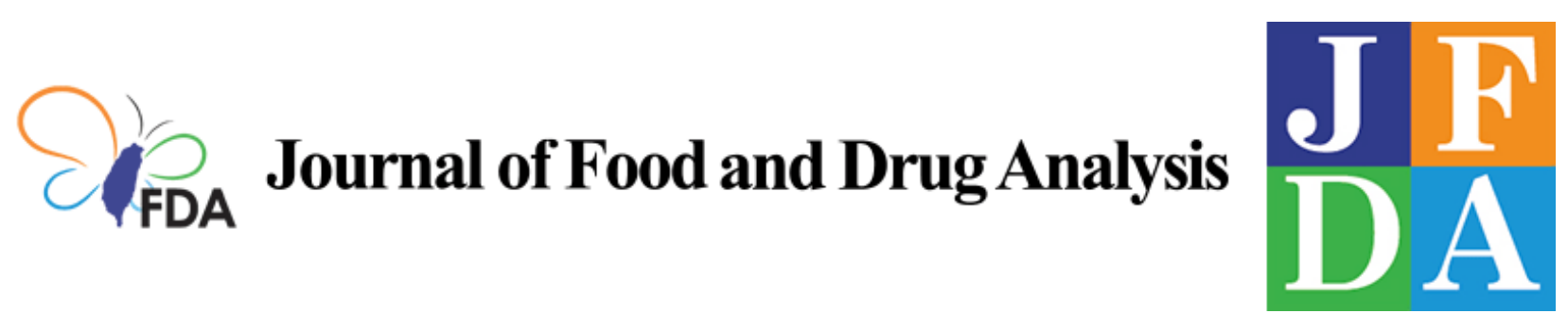

Volume 29 | Issue 3

Article 6

2021

\title{
An extractive nanoelectrospray ionization-mass spectrometry method for Chinese herbal medicine authentication
}

Follow this and additional works at: https://www.jfda-online.com/journal

Part of the Food Science Commons, Medicinal Chemistry and Pharmaceutics Commons, Pharmacology Commons, and the Toxicology Commons

(c) (i) (9)

This work is licensed under a Creative Commons Attribution-Noncommercial-No Derivative Works 4.0 License.

\section{Recommended Citation}

Lee, Chia-Hsin; Huang, Hui-Chi; Tseng, Mei-Chun; and Chen, Chao-Jung (2021) "An extractive nanoelectrospray ionization-mass spectrometry method for Chinese herbal medicine authentication," Journal of Food and Drug Analysis: Vol. 29 : Iss. 3 , Article 6.

Available at: https://doi.org/10.38212/2224-6614.3368

This Original Article is brought to you for free and open access by Journal of Food and Drug Analysis. It has been accepted for inclusion in Journal of Food and Drug Analysis by an authorized editor of Journal of Food and Drug Analysis. 


\title{
An extractive nanoelectrospray ionization-mass spectrometry method for Chinese herbal medicine authentication
}

\author{
Chia-Hsin Lee ${ }^{a}$, Hui-Chi Huang ${ }^{a, b}$, Mei-Chun Tseng ${ }^{c}$, Chao-Jung Chen ${ }^{\mathrm{d}, e, *}$ \\ ${ }^{a}$ Department of Chinese Pharmaceutical Sciences and Chinese Medicine Resources, China Medical University, Taichung 40402, Taiwan \\ ${ }^{\mathrm{b}}$ Master Program for Food and Drug Safety, China Medical University, Taichung 40402, Taiwan \\ ${ }^{\mathrm{c}}$ Institute of Chemistry, Academia Sinica, Taipei 11529, Taiwan \\ d Proteomics Core Laboratory, Department of Medical Research, China Medical University Hospital, Taichung 40447, Taiwan \\ ${ }^{\mathrm{e}}$ Graduate Institute of Integrated Medicine, China Medical University, Taichung 40402, Taiwan
}

\begin{abstract}
Herein, we describe a rapid, sensitive, and nondestructive method-extractive nanoelectrospray ionization-mass spectrometry (EnESI-MS) - for traditional Chinese medicine (TCM) authentication. The mass-spectral fingerprints of volatile compounds released from various TCMs can be rapidly acquired using EnESI-MS without sample pretreatment. EnESI-MS was applied to successfully differentiate between two commonly used medicinal herbs, Schisandra chinensis and Schisandra sphenanthera, which are morphologically similar but exhibit different therapeutic effects. Specific volatile compounds of each herb in a ten-component Chinese herbal product, Jia Wei Xiao Yao San, were also identified, and the method was applied to discriminate between the commercial product and a substandard version.
\end{abstract}

Keywords: Authentication, Extractive nanospray ionization, Traditional Chinese medicine, Volatile compounds

\section{Introduction}

$\mathrm{T}$ raditional Chinese medicine (TCM) has been practiced for thousands of years. In the 21st century, TCM has been widely studied for drug discovery [1-4]. Since the quality of a medicine may influence its efficacy and safety, quality control is essential. However, a major concern for quality control in TCM is the misidentification of herbal species, which can occur inadvertently because of misidentification due to morphological similarity, multiple sources, or confusing naming conventions (differing regional names for the same herbs, or different herbs with the same names from different regions), or intentionally, through product counterfeiting. Consequently, the authentication of TCMs is crucial to ensure their efficacy and safety. Several identification methods are widely used to authenticate TCMs, based on morphological, microscopic, chemical, and/or genetic examination [5]. More recently, other approaches have been developed for rapid TCM identification, such as cysteine-rich peptide (CRP) fingerprinting [6] and electronic nose (Enose) systems [7]. E-nose technology benefits from non-destructive sample preparation, relatively fast assessment, and real-time information, which facilitates the monitoring of diverse volatile components. Because odor can be an important property of TCMs, E-noses have also been applied

Abbreviations: TCM, Traditional Chinese medicine; E-nose, Electronic nose; ESI-MS, Electrospray ionization-mass spectrometry; EESI, Extractive electrospray ionization; EnESI, Extractive nanoelectrospray ionization source nanospray ion source; S. chinensis, Schisandra chinensis; S. sphenanthera, Schisandra Sphenanthera; PCA, Principal component analysis,

Received 6 March 2021; revised 16 May 2021; accepted 22 June 2021.

Available online 15 September 2021.

* Corresponding author: Proteomics Core Laboratory, Department of Medical Research, China Medical University Hospital, Taichung 40447, Taiwan. Fax: 886-4-22037690.

E-mail address: cjchen@mail.cmu.edu.tw (C.-J. Chen). 
their analyses [7,8]. However, an E-nose system may be limited in TCM identification because of its unsatisfactory recognition accuracy and lack of chemical identification information [8].

Electrospray ionization-mass spectrometry (ESIMS) is a sensitive, specific, and highly reliable analytical technique for detecting chemical compounds in the solution phase. In ESI-MS analysis, dissolved analytes are infused directly into the instrument to generate ions, which may cause significant ion suppression effects and lead to a low tolerance against sample matrixes. Therefore, samples with complex matrices must undergo pretreatments such as purification, extraction, and chromatographic separation before MS analysis, to lower the ion suppression effect and increase sensitivity. However, tedious sample preparation usually makes the analytical work laborious and timeconsuming. In 2006, Cooks et al. developed another ionization method, extractive electrospray ionization (EESI) [9], that uses an ESI sprayer and a sample sprayer to produce charged microdroplets of solvent and nebulize the sample, respectively. During the EESI process, sample droplets from the sample sprayer are initially aerosolized and then ionized by the charged solvent droplets sprayed by the ESI spray when they intersect [10]. In 2007, the EESI interface was slightly modified by Zenobi et al. by using air as a carrier gas to introduce gaseous analytes into the electrospray beam to monitor the maturity of fruits [11]. This made EESI an alternative ionization method for the analysis of samples with complex matrices, and has been applied in the analysis of breath aerosols [12], reaction mixtures [13], and toxic substances from biological samples [14]. However, EESI-MS has not been applied for the analysis of the volatile components of TCMs.

In this study, to develop a simple, sensitive, practical MS platform for the rapid detection of volatile compounds and apply it to TCM authentication, a nanoESI source was modified as an extractive nanoelectrospray ionization source and coupled to a highresolution mass spectrometer (EnESI-MS). Comparing with a hard ionization technique of EIMS, the soft ionization technique of EnESI-MS can produce the intact molecular ions in MS scan mode without generating fragmented ions, which cause ambiguous and complex mass spectrum. In addition, without the use of heating injector and GC, EnESI-MS is more feasible to analyze thermal labile compounds and is a more rapid and easily handling method compared to GC-EI-MS. To the best of our knowledge, it is the first study to develop an extractive nanoelectrospray ionization source (EnESI) with higher sensitivity compared to EESI. The EnESI-MS was applied to analyze two TCMs, Wu Wei Zi and Jia Wei Xiao Yao San. Wu Wei Zi consists of two closely related species, Bei Wu Wei (Schisandra chinensis (S. chinensis)) and Nan Wu Wei (Schisandra Sphenanthera (Schisandra sphenanthera)), which have quite similar morphologies; a well-experienced examiner is needed for morphological identification. Because they have dissimilar therapeutic effects, differentiation between the S. chinensis and S. sphenanthera fruits is important for clinical efficacy. Jia Wei Xiao Yao San, composed of ten herbs, is a traditional formula to soothe the liver and fortify the spleen [15] and is the most frequently prescribed formula in Taiwan for the treatment of agitation, functional dyspepsia, insomnia and mood disorders [16]. Therefore, the EnESI-MS approach was applied to discover marker peaks for Wu Wei $\mathrm{Zi}$ and the ten herbs of Jia Wei Xiao Yao San, to evaluate its potential use in TCM authentication.

\section{Materials and methods}

\subsection{Reagents and chemicals}

Acetonitrile (ACN) was purchased from J.T. Baker (Phillipsburg, NJ, USA). Deionized water (Milli-Q, Millipore, USA) was used. Fruits of $S$. chinensis and S. sphenanthera, Moutan Radicis Cortex, Angelicae Sinensis Radix, Menthae herba, Atractylodis Macrocephalae Rhizoma, Gardeniae Fructus, Bupleuri Radix, Paeoniae Alba Radix, Poria, Zingiberis Rhizoma, and Glycyrrhizae Preparata Radix were all purchased from three different stores. (Lian-He, Taichung, Taiwan; Yi-De, Taichung, Taiwan; JiingHan-Tang, New Taipei city, Taiwan) Jia Wei Xiao Yao San were purchased from Sheng Chang Pharmaceutical Co., Ltd. (Taoyuan, Taiwan) and SunTen Pharmaceutical Co., Ltd. (Taichung, Taiwan). Home-made Jia Wei Xiao Yao San powder was prepared according to the method for concentrated TCMs from the Taiwan Herbal Pharmacopeia(3rd Edition) [15]. The ten herbs with the following proportions were mixed: Moutan Radicis Cortex $(1.25 \mathrm{~g})$, Angelicae Sinensis Radix (2 g), Menthae herba (1 g), Atractylodis Macrocephalae Rhizoma (2 g), Gardeniae Fructus (1.25 g), Bupleuri Radix (2 g), Paeoniae Alba Radix (2 g), Poria (2 g), Zingiberis Rhizoma ( $2 \mathrm{~g})$, and Glycyrrhizae Preparata Radix ( $1 \mathrm{~g})$. The herbal mixture was grinded using an electrical blade grinder to have a tiny powder. The powder of herb mixture was stored in a tightly closed bottle to prevent the loss of volatile compounds, and a tea bag was used to packed the powder (2g) for EnESI-MS analysis. 


\subsection{EESI and EnESI-MS analysis}

Samples were placed in a clean, dry glass container to avoid potential chemical contamination. We modified our ESI (Bruker, Germany) and nanoESI sources (CaptiveSpray, Bruker, Germany) as EESI and an EnESI sources, respectively. For either EESI or EnESI, a tube, originally used as a nebulization gas tube in ESI or nanoESI, was connected to the sample container. For EESI, $\mathrm{N}_{2}$ ( $10 \mathrm{psi}$ ) was infused into the sample container and the volatile compounds were delivered from the samples into the EESI source. An $80 \% \mathrm{ACN} /$ water mixture was infused as the ESI solution at $4 \mu \mathrm{L} / \mathrm{min}$ using a syringe pump (KD Scientific Inc, MA). The spray voltage was operated at $3600 \mathrm{~V}$. For EnESI, $\mathrm{N}_{2}$ ( $\sim 4$ psi) was infused into the sample container and the volatile compounds were delivered from the sample into the EnESI source. An $80 \% \mathrm{ACN} /$ water mixture was infused as the nanoESI solution at $300 \mathrm{~nL} / \mathrm{min}$ by a nanoflow pump (Ultimate 3000, Dionex). The spray voltage was operated at $2100 \mathrm{~V}$. EESI and EnESI were performed using a QTOF-MS instrument (Maxis Impact QTOF mass spectrometer, Bruker Daltonics). Data were collected in the positive ion mode. Precursor ions were isolated with 3-4 Da. Spectra were collected for $1.5 \mathrm{~min}$ and processed (DataAnalysis, Bruker, Germany).

\subsection{Processing of MS spectral data}

The raw data from the mass peak intensity lists were exported manually as .csv files and compressed to .zip files. The compressed files were loaded into MetaboAnalyst (www. metaboanalyst.ca) for PCA analysis. Mass tolerance was set at $0.025 \mathrm{~m} / \mathrm{z}$, and no normalization or data transformation methods were used.

\section{Results and discussion}

\subsection{EnESI optimization}

Fig. 1 shows the flow direction of the gas and spray solution inside the EnESI. Volatile compounds from the sample bottle are swept by $\mathrm{N}_{2}$ into the EnESI for analysis. An EESI sprayer ion source is generated by introducing volatile analytes into the electrospray beam through the desolvation gas line. The sensitivities of EESI and EnESI were compared for the volatiles in S. chinensis. As shown in Fig. 2a, EESI detects volatile compounds with $\mathrm{m} / \mathrm{z}$ 97.03, 111.04, 121.10, 147.04, 205.19, and 219.17 from $S$. chinensis ( 1.5 g), compared to the background signals from an empty bottle (Fig. 2b). The peak of $\mathrm{m} / \mathrm{z}$ 147.04 could be coumarin due to their ESI-MS/MS spectra (Fig. S1) in a reported study [17]. EnESI also detects the aforementioned volatile compounds from S. chinensis (Fig. 2c); background signals (an empty bottle) are presented in Fig. 2 d.

However, compared to EESI, EnESI shows a significant signal improvement for these volatile compounds and has a 119 folds higher peak intensity of $\mathrm{m} / \mathrm{z} 111.04$ (intensity $2.5 \times 10^{6}$, S/N 7733 in EnESI vs. intensity $2.1 \times 10^{4}$, S/N 33.5 in EESI), 97 folds higher peak intensity of $\mathrm{m} / \mathrm{z} 121.01$ (intensity $8.0 \times 10^{5}, \mathrm{~S} / \mathrm{N}$ 2406.7 in EnESI vs. intensity $8.2 \times 10^{3}$, S/N 17.9 in EESI), 110 folds higher peak intensity of $\mathrm{m} / z 147.04$ (intensity $1.1 \times 10^{6}, \mathrm{~S} / \mathrm{N} 2965.8$ in EnESI vs. intensity $1.0 \times 10^{4}, \mathrm{~S} / \mathrm{N} 15.6$ in EESI) and 340 folds higher peak intensity of $\mathrm{m} / z 205.19$ (intensity $1.7 \times 10^{6}$, S/N 3774.4 in EnESI vs. intensity $5.0 \times 10^{3}, \mathrm{~S} / \mathrm{N} 11.1$ in EESI). In EESI, a Taylor cone is formed at the spray

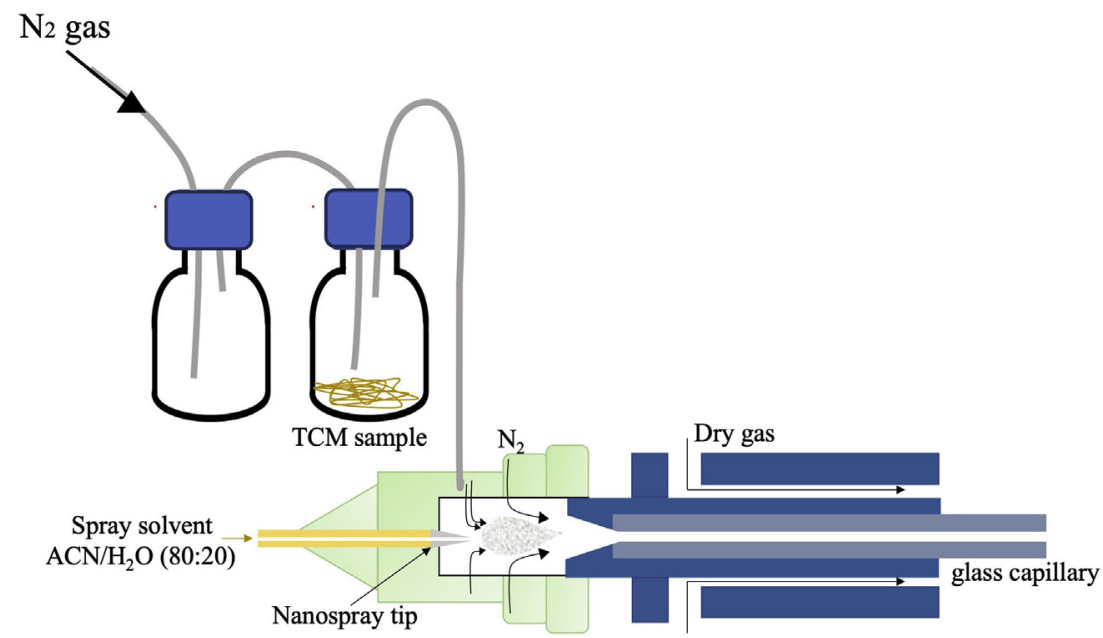

Fig. 1. Schematic diagram of EnESI. 
(a)

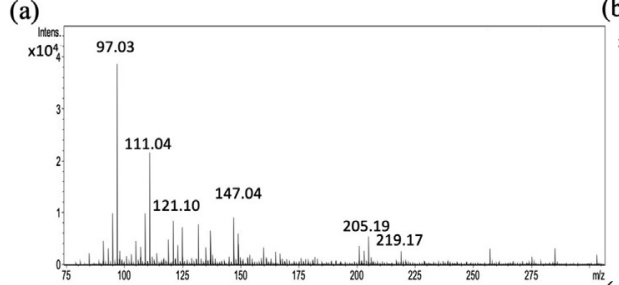

(d)

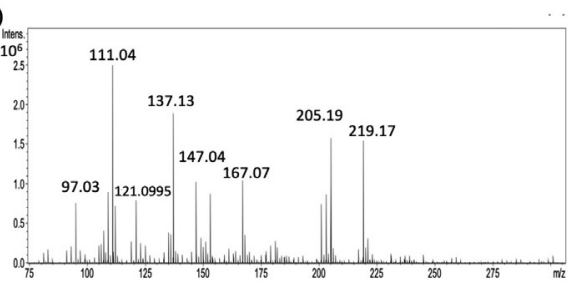

(b)

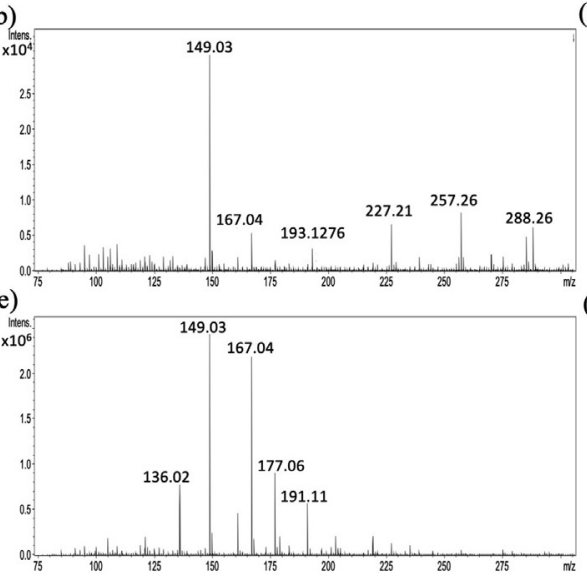

(c)
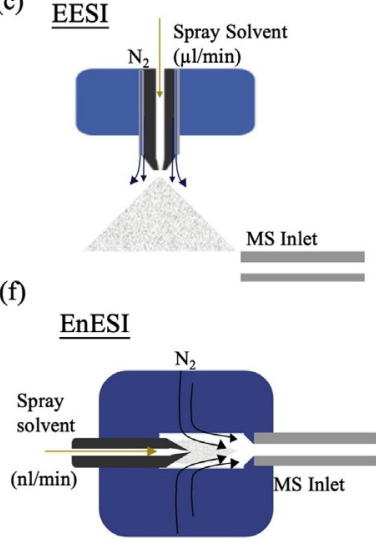

Fig. 2. Sensitivity comparison between EESI and EnESI using S. chinensis ( 1.5 g). (a) Volatile compound fingerprints of S. chinensis by EESI. (b) Blank signals obtained from an empty bottle with EESI. (c) Schematic diagram of EESI. (d) Volatile compound fingerprints of S. chinensis by EnESI. (e) Blank signals obtained from an empty bottle with EnESI. (f) Schematic diagram of EnESI.

tip and emits a fine mist of droplets into an open field (Fig. 2c). Analytes are scattered so that not all are extracted in the solvent and carried into the MS inlet. In EnESI, gaseous analytes sweep around the spray tip and focus the Taylor cone into the MS in a small, closed space (Fig. 2f). This design may increase the extraction efficiency for volatile compounds by the spray solution, resulting in high sensitivity for volatile compound analysis. Because volatile organic compounds are compounds that have a high vapor pressure and high nonpolar property, a high percentage of ACN may be suitable for the extraction of these non-polar ions in EnESI. Different percentages $(5 \%, 50 \%$ and $80 \%)$ of ACN were tested, and the use of $80 \% \mathrm{ACN}$ were found to have more peaks in a larger $m / z$ range $(\sim m / z 280-\mathrm{m} /$ z 460). (Fig. S2) Therefore, $80 \%$ ACN was selected for the spray solvent composition in EnESI.

\subsection{Concentration-dependent signal intensity}

As shown in Fig. 3, the volatiles in S. chinensis samples as small as 1 grain $(\sim 65 \mathrm{mg})$ can be detected by EnESI, and the intensities of the $m / z 111.04$ peak increase in a linear fashion when the sample loading increases from 1 to 9 ( 550 $\mathrm{mg})$ grains. However, the intensity of the $\mathrm{m} / \mathrm{z} 111.04$ peak decreases for 12 $(\sim 728 \mathrm{mg})$ and 15 grains $(\sim 910 \mathrm{mg})$ of S. chinensis. This may be due to the ion suppression effect of the $\mathrm{m} / \mathrm{z} 111.04$ peak by other ions, because the $\mathrm{m} / \mathrm{z}$ 147.04. and 167.07 peaks are of higher intensity than $\mathrm{m} / \mathrm{z} 111.04$ at 12 and 15 grains (data not shown). This may indicate that the signals for volatile compounds in EnESI are concentration-dependent and may be applicable for material quantification.

\subsection{Differentiation between $S$. chinensis and $S$. sphenanthera}

Because Bei Wu Wei (S. chinensis) and Nan Wu Wei (S. sphenanthera) are difficult to distinguish based on appearance, the two herbal species were analyzed by EnESI-MS to identify specific identifying markers. Samples of both species were purchased from three different stores and subjected to analysis ( $2 \mathrm{~g})$; the raw data sets were imported to MetaboAnalyst for PCA analysis. As shown in Fig. 4a, the MS signals from $S$. chinensis and $S$. sphenanthera can be separated into two distinct groups, indicating some differences in chemical constituents. The corresponding loading plot (Fig. 4b) shows that the peaks at $\mathrm{m} / \mathrm{z} 111.04$ and 153.13 are the most abundant peaks in S. chinensis, whereas that at $m / z 167.07$ is predominant in $S$. sphenanthera. The most abundant peaks of $S$. chinensis and S. sphenanthera from PCA analysis are consistent with the peaks observed in their EnESI-

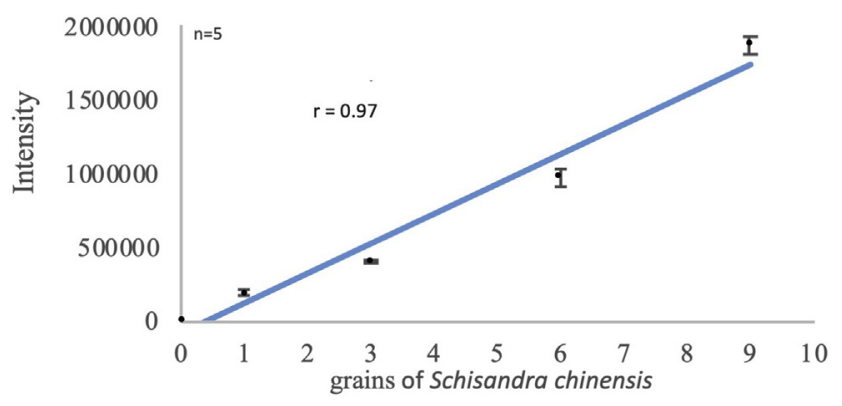

Fig. 3. EnESI-MS peak signals of $m / z 111.04$ for 1 to 9 grains of $S$. chinensis $(n=3)$. 
(a)

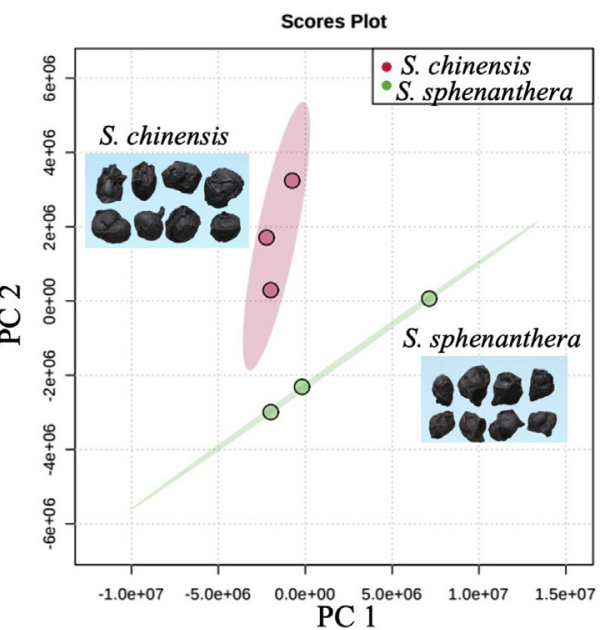

(c)

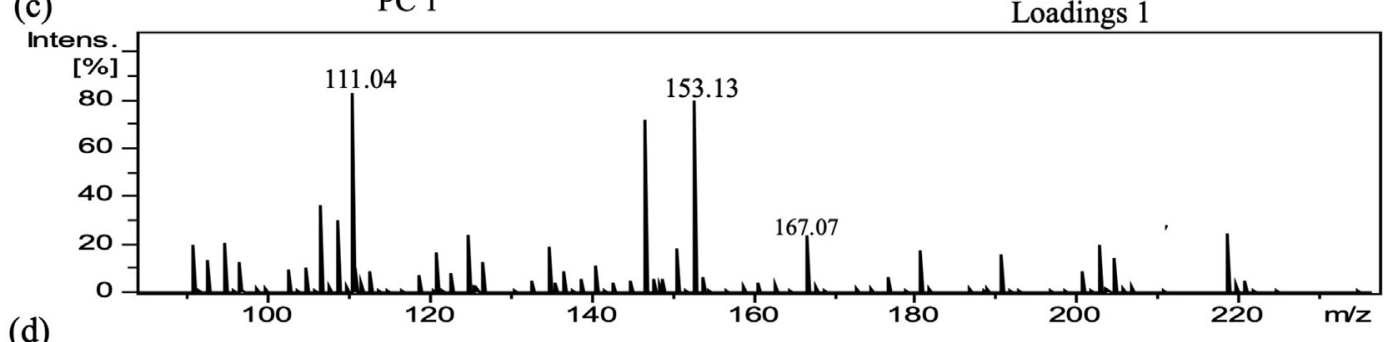

(d)

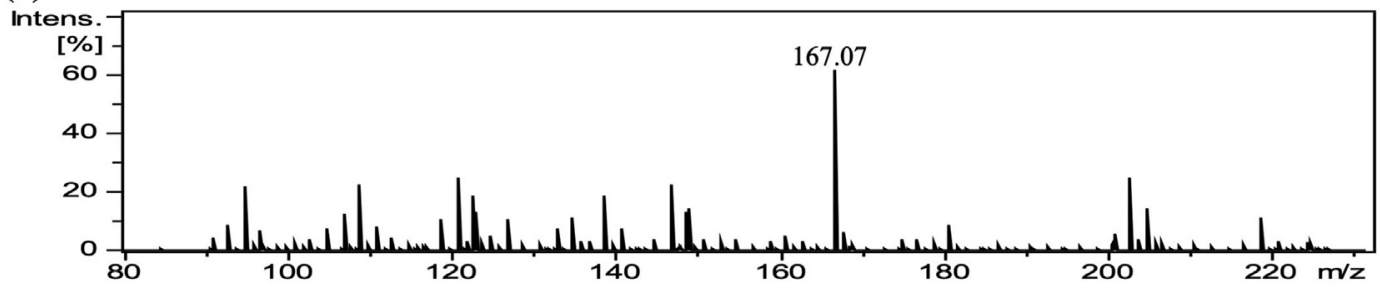

Fig. 4. (a) PCA results for S. chinensis $(n=3)$ and S. sphenanthera $(n=3)$. (b) Loading plot of PC1 and PC2 components. EnESI-MS spectra of (c) S. chinensis and (d) S. sphenanthera.

QTOF-MS spectra (Fig. 4c, d). Therefore, peaks at $m$ / $z 111.04,153.13$, and 167.07 can be used as marker peaks to differentiate between the two species. The peak of $\mathrm{m} / \mathrm{z} 167.07$ can be identified as paeonol based on their matched ESI-MS/MS spectra (Fig. S3) in a reported study [18]. However, the other two peaks cannot be identified due to the absence of matched MS/MS spectra in mass spectral databases.

\subsection{Marker peak determination for ten herbs in Jia Wei Xiao Yao San}

To determine markers for the identification of Jia Wei Xiao Yao San, ten commonly used raw herbal components were bought from three different stores and analyzed using EnESI-MS. Their morphologies are shown in Fig. S4. Principal component analysis (PCA) and a loading plot were used to analyze the possible marker peaks of Angelicae Sinensis Radix. As shown in Fig. 5a, EnESI-MS signals from this herb and the blank (empty bottle) can be separated

(b)

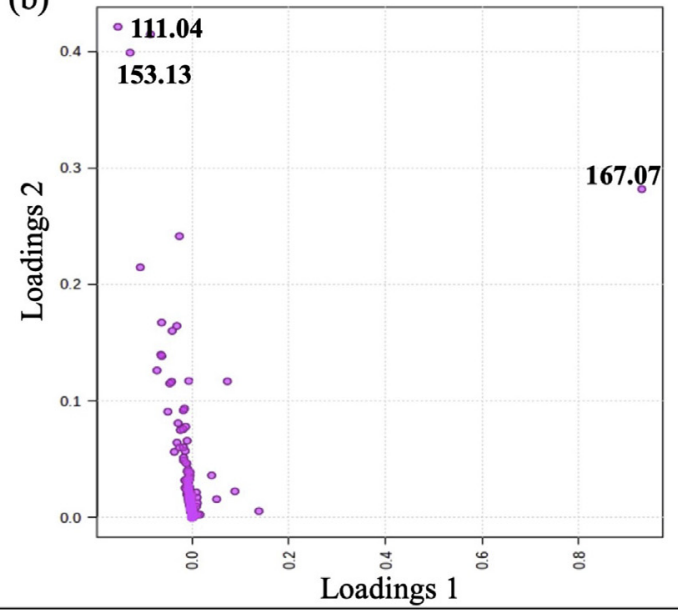

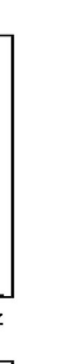


(a)

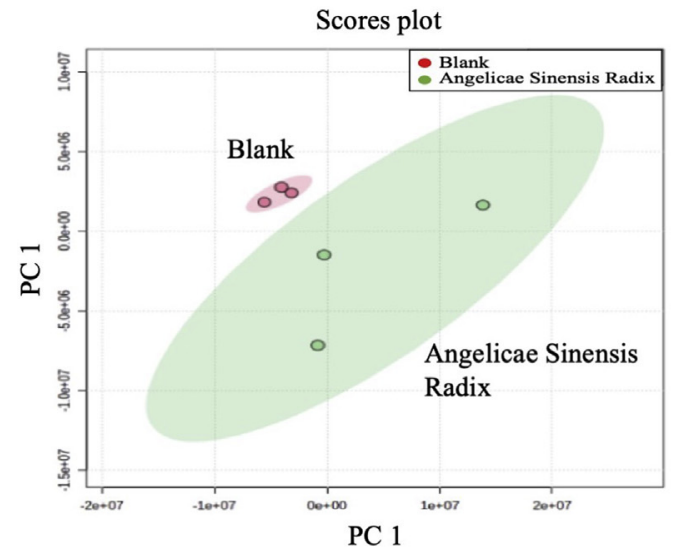

(b)

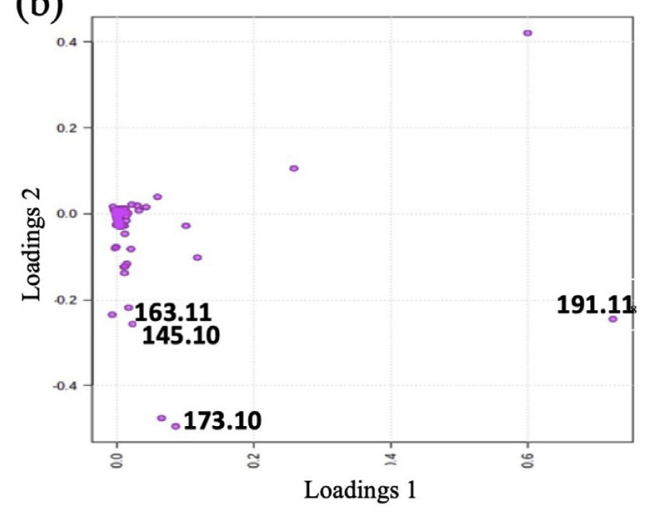

(c)

(d)
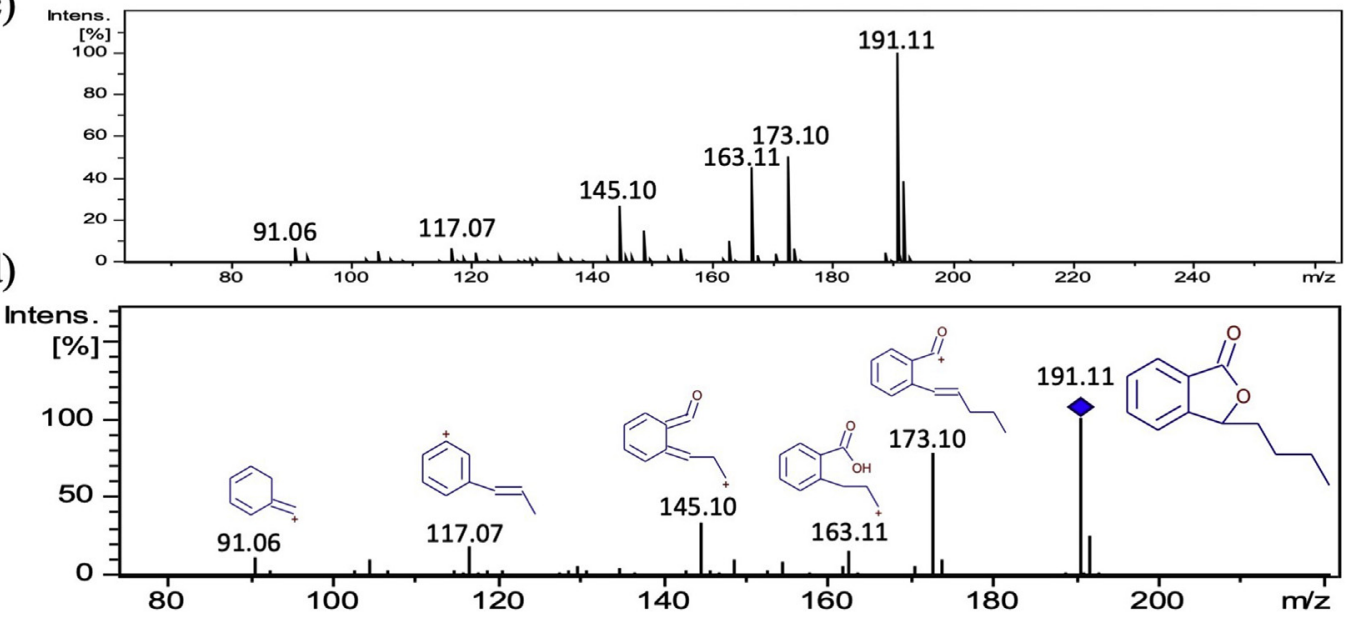

Fig. 5. (a) PCA result for Angelicae Sinensis Radix $(n=3)$ and (b) loading plot of PC1 and PC2 components. (c) EnESI-MS spectrum of Angelicae Sinensis Radix and (d) MS/MS spectrum of the marker peak at $\mathrm{m} / \mathrm{z}$ 191.11. The fragments of structural elucidation were obtained by using software Mass Frontier 8.0 (Thermo Fisher Scientific, Waltham, United States).

Table S1 presents the marker peaks for the ten herbs in Jia Wei Xiao Yao San detected by EnESIMS, as well as their fragment ions with possible compound identities. Only two marker peak compounds, butylphthalide in Angelicae Sinensis Radix and paeonol in Moutan Radicis Cortex [18,21], could be identified based on their matched ESI-MS/MS spectra in reported studies [18-21]. Because most volatile compound were analyzed with GC-MS, these volatile compounds only have EI-MS spectra in MS spectra databases. Thurman, E. M. et al. compared EI with ESI spectra, and found that although EI and ESI spectra are different, they may still have some fragmented ions in common [22]. Therefore, the other marker peaks with no available ESI-MS/MS spectrum in database were referred to their correspond EI spectra in NIST database or reported studies. The potential structures identified from EI-MS spectra were further matched to the theoretical ESI-MS fragments elucidated by Mass Frontier software (version 8.0, Thermo Fisher Scientific, Waltham, United States). Finally, atractylon was identified as the marker peak $(\mathrm{m} / \mathrm{z} 217.16)$ of Atractylodis Macrophalae Rhizoma; 2,6,6-trimethyl1,3-cyclohexadiene-1-carboxaldehyde was identified as the marker peak $(\mathrm{m} / \mathrm{z}$ 151.11) of Gardeniae Fructus; Eucalyptol was identified as the marker peak ( $m / z$ 155.10) of Bupleuri Radix [23]; 2-pentylfuran was identified as the marker peak $(\mathrm{m} / \mathrm{z}$ 139.11) of Poria. The marker peak of $m / z 153.13$ were identified as four possible compounds (Cyclohexanone, 5methyl-2-(1-methylethylidene)-; (E)-3(10)-Caren-4ol; 2-Cyclohexen-1-ol, 1-methyl-4-(1-methylethenyl)-, trans-; thujone), because they have the same theoretical $[\mathrm{M}+\mathrm{H}]^{+}$value and the same elucidated fragmented ions, and have been identified in a GC-MS study of Mentha haplocalyx [24] (Table S1). Because the volatile compounds were not separated in EnESI-MS, the MS/MS spectrum may contain all the fragment ions from the 4 possible compounds. However, the structures of marker peaks of 121.1 (paeoniae alba radix), 137.13 (zingiberis rhizome) and 100.07 (glycyrrhizae preparata radiz) cannot be identified in this study. 


\subsection{EnESI-MS analysis of Jia Wei Xiao Yao San raw herb powder mixture and commercial products}

To evaluate the performance of EnESI-MS in the authenticity of Jia Wei Xiao Yao San, the homemade and commercial Jia Wei Xiao Yao San products were analyzed and compared. The marker peaks of each herb (Table S1) are found in the MS spectrum of the home-made preparation (Fig. 6a): Atractylodis Macrocephalae Rhizoma (marker peak: $m / z$ 217.16), Angelicae Sinensis Radix ( $m / z$ 191.11),
Moutan Radicis Cortex ( $m / z$ 167.07), Menthae Herba $(\mathrm{m} / \mathrm{z}$ 153.13), Gardeniae Fructus $(\mathrm{m} / \mathrm{z}$ 151.11), Bupleuri Radix ( $m / z$ 155.10), Poria $(\mathrm{m} / \mathrm{z}$ 139.11), Paeoniae Alba Radix ( $m / z$ 121.10), Zingiberis Rhizoma $(\mathrm{m} / \mathrm{z}$ 137.13), and Glycyrrhizae Preparata Radix $(m / z$ 100.07).

The aforementioned marker peaks of the herbs in the home-made sample are also observed in the MS spectrum of a commercial Jia Wei Xiao Yao San product purchased from a TCM company (Sheng Chang Pharmaceutical Co., Fig. 6b). The EnESI-MS

(a)

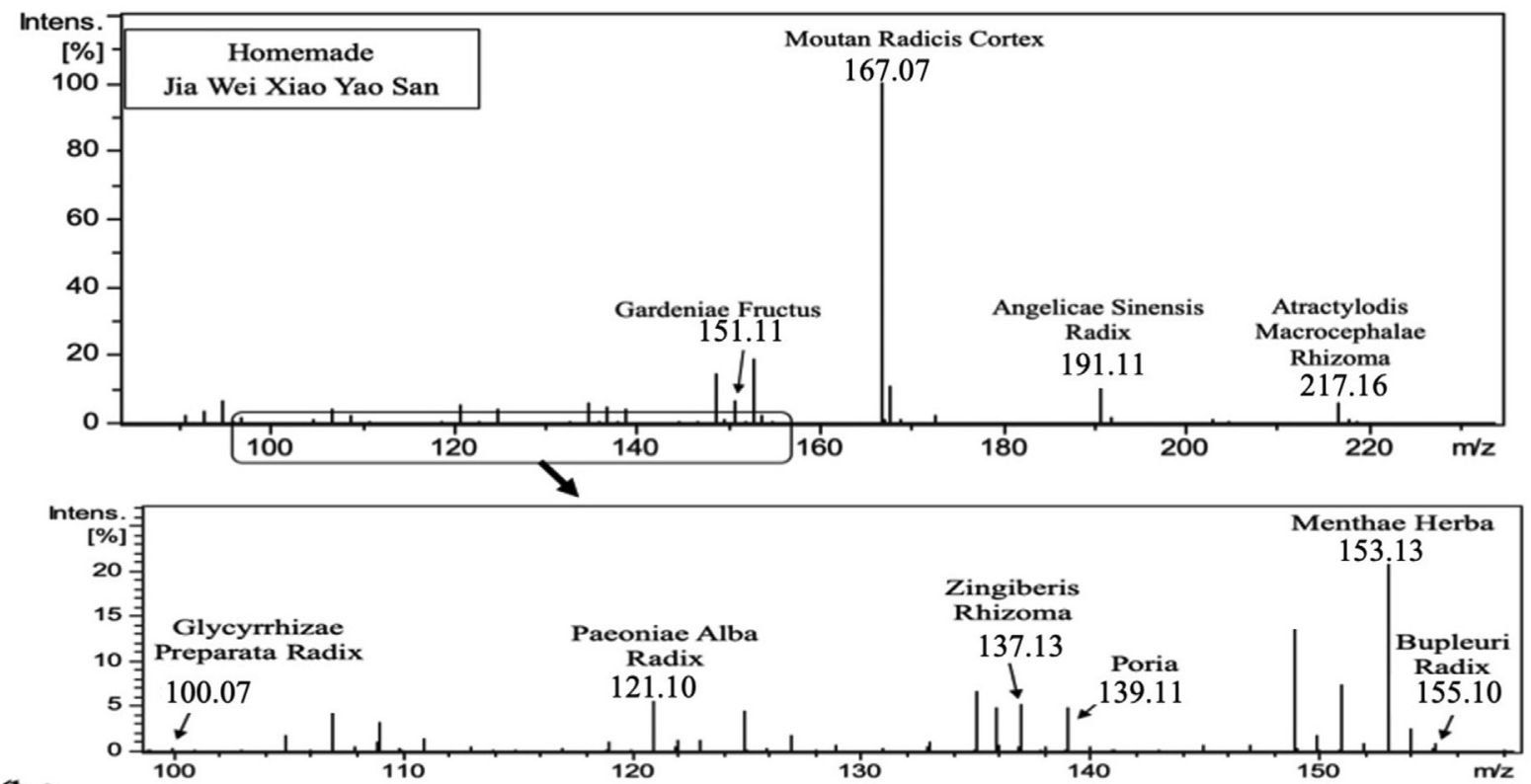

(b)

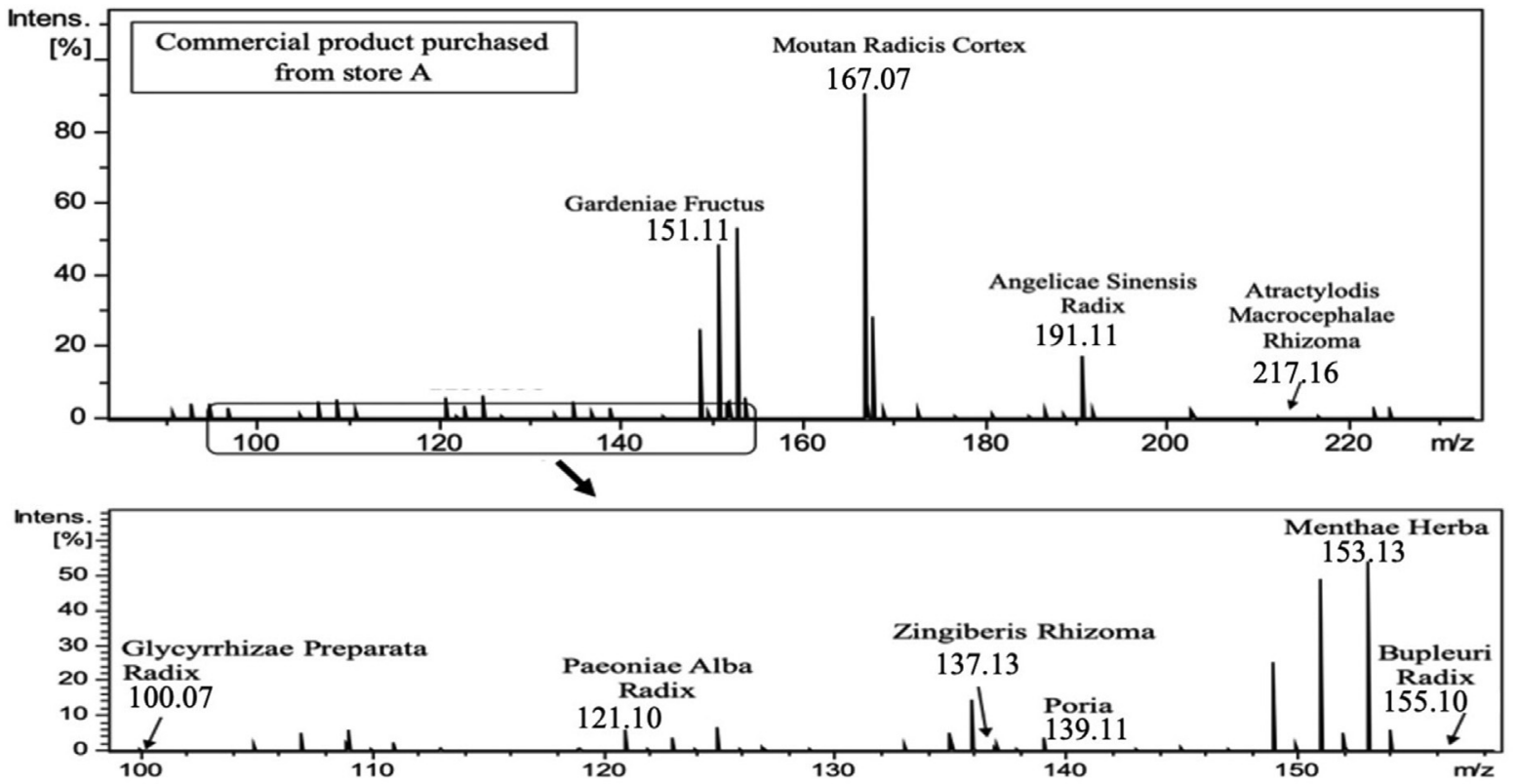

Fig. 6. EnESI-MS spectra of Jia Wei Xiao Yao San from (a) the homemade powder and (b) a commercial product. 
(a)
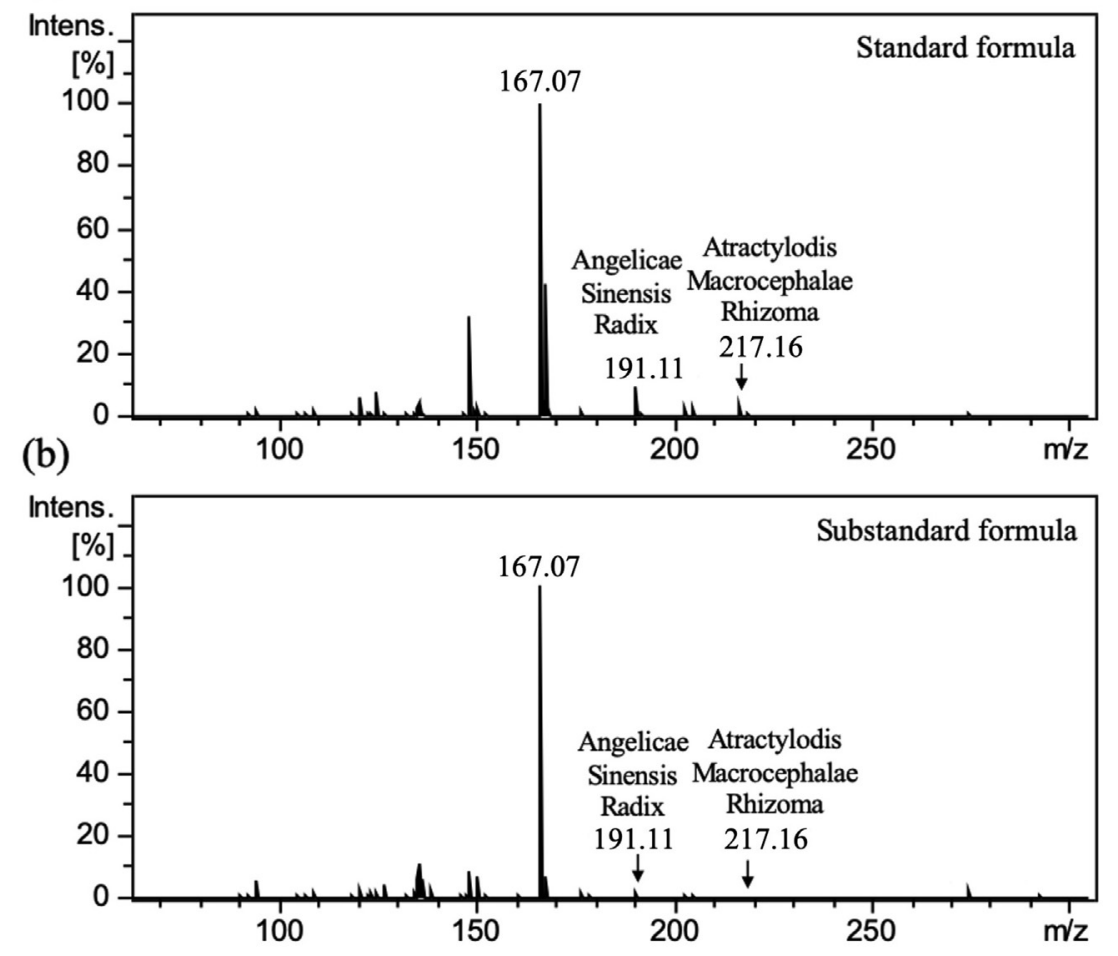

Fig. 7. EnESI-MS spectrum of (a) a standard preparation of Jia Wei Xiao Yao San powder and a (b) modified formula.

analysis of another commercial Jia Wei Xiao Yao San product, purchased from another supplier (Sun-Ten Pharmaceutical Co.), is shown in Fig. S14. The EnESI-MS spectra of the two commercial products are similar to our homemade powder mixture. The LOD of Jia Wei Xiao Yao San by EnESI-MS was found to be $\sim 20 \mathrm{mg}$ by evaluating the two lowest marker peaks of $m / z 100.07$ (S/N: 3.9) and $m / z 155.10$ (S/N: 3.6). Therefore, EnESI-MS could be a rapid and attractive method for the quality control of commercial concentrated products.

\subsection{EnESI-MS for the determination of substandard TCM products}

Substandard TCM products have been a serious issue that influence safety and efficacy. Substandard products can arise for a variety of reasons, such as improper storage methods that cause drug deterioration, the inclusion of contaminants, or out-ofspecification drug quality or quantities. Therefore, the ability of EnESI-MS to identify substandard TCM products was next assessed. A modified Jia Wei Xiao Yao San formula was prepared by decreasing the amounts of Angelicae Sinensis Radix and Atractylodis Macrocephalae Rhizoma to a half and a quarter of their original quantities, respectively. As shown in Fig. 7, compared to the original formula (Fig. 7a), the marker peak intensities for Angelicae Sinensis Radix and Atractylodis Macrocephalae Rhizoma significantly decrease in the modified formula (Fig. 7b). The degrees of decrease were quantified by comparing the peak intensities of the markers for these two herbs to the most abundant peak for the standard formula, $m / z$ 167.07. The $m / z$ 191.11/167.07 value decreases from 0.094 to 0.021 when the amount of Angelicae Sinensis Radix is reduced by $50 \%$. The $\mathrm{m} / \mathrm{z} 217.16 / 167.07$ value changes from 0.041 to 0.008 for the $75 \%$ reduction in Atractylodis Macrocephalae Rhizoma content. Therefore, EnESI-MS may be able to detect proportional changes in the constituents of an herbal mixture and be applied in the quality control of commercial concentrated products.

\section{Conclusion}

In this study, a sensitive EnESI-MS method was developed for the rapid analysis of volatile compounds in traditional Chinese medicines. The EnESI-MS signals for the volatile compounds increased with the amount of material. EnESI-MS was applied to successfully differentiate $S$. chinensis from S. sphenanthera. Marker peaks of ten herbs in Jia Wei Xiao Yao San were also identified and applied for the identification of commercial Jia Wei 
Xiao Yao San and a substandard formulation. EnESI-MS could be a practical and attractive method for the rapid authentication of TCMs and the quality control of commercial concentrated TCM products.

\section{Conflicts of interest}

The authors declare no conflict of interest.

\section{Acknowledgments}

This work was supported by grants from the China Medical University, Taiwan (CMU-108-MF04) and the Ministry of Science and Technology, Taiwan, China (MOST 109-2113-M-039-001).

\section{Supporting information.}

Supplementary Table 1. Marker peaks for ten herbs in Jia Wei Xiao Yao San.

\begin{tabular}{|c|c|c|c|c|}
\hline & Herb & $\begin{array}{l}{[\mathrm{M}+\mathrm{H}]^{+} \text {of }} \\
\text { marker peak }\end{array}$ & $\begin{array}{l}\text { Predominant peaks in } \\
\text { MS/MS (relative intensity) }\end{array}$ & Possible identity \\
\hline 1. & Angelicae Sinensis Radix & 191.11 & $\begin{array}{l}191.11(100) \\
173.10(78) \\
163.11(20) \\
145.10(34) \\
117.07(25) \\
91.06(18)\end{array}$ & Butylphthalide \\
\hline 2. & Moutan Radicis Cortex & 167.07 & $\begin{array}{l}167.07(100) \\
149.06(64) \\
121.06(58)\end{array}$ & Paeonol \\
\hline 3. & $\begin{array}{l}\text { Atractylodis Macrocephalae } \\
\text { Rhizoma }\end{array}$ & 217.16 & $\begin{array}{l}217.16(100) \\
199.15(11.3) \\
161.10(10) \\
147.12(9.3) \\
133.10(6) \\
121.10(5) \\
107.10(4) \\
95.05(6)\end{array}$ & Atractylon \\
\hline 4. & Gardeniae Fructus & 151.11 & $\begin{array}{l}151.11(100) \\
135.12(20) \\
123.1(15) \\
109.10(23) \\
95.1(24) \\
93.07(19)\end{array}$ & $\begin{array}{l}\text { 2,6,6-Trimethyl-1, } \\
\text { 3-Cyclohexadiene-1-Carboxaldehyde }\end{array}$ \\
\hline 5. & $x^{2}$ & 155.10 & $\begin{array}{l}139.08(10) \\
137.09(10) \\
109.10(100) \\
95.08(29) \\
93.06(23.8)\end{array}$ & Eucalyptol \\
\hline 6. & Poria & 139.11 & $\begin{array}{l}139.11(100) \\
121.10(9.3) \\
97.06(9.0) \\
95.08(9.0)\end{array}$ & 2-Pentylfuran \\
\hline 7. & Menthae Herba & 153.13 & $\begin{array}{l}153.13(100) \\
135.1(20) \\
111.08(20) \\
109.11(30) \\
107.08(30) \\
97.06(38) \\
95.07(20) \\
93.07(47)\end{array}$ & $\begin{array}{l}\text { (1) Cyclohexanone, } \\
\text { 5-methyl-2-(1-methylethylidene)- } \\
\text { (2) (E)-3 (10)-Caren-4-ol } \\
\text { (3) 2-Cyclohexen-1-ol, } \\
\text { 1-methyl-4-(1-methylethenyl)-, } \\
\text { trans-(4) Thujone }\end{array}$ \\
\hline 8. & Paeoniae Alba Radix & 121.10 & $\begin{array}{l}121.10(100) \\
91.05(31.8)\end{array}$ & Not identified \\
\hline 9. & Zingiberis Rhizoma & 137.13 & $\begin{array}{l}137.13(100) \\
107.09(99) \\
95.09(98)\end{array}$ & Not identified \\
\hline 10. & Glycyrrhizae Preparata Radix & 100.07 & $\begin{array}{l}100.07(100) \\
97.06(4)\end{array}$ & Not identified \\
\hline
\end{tabular}




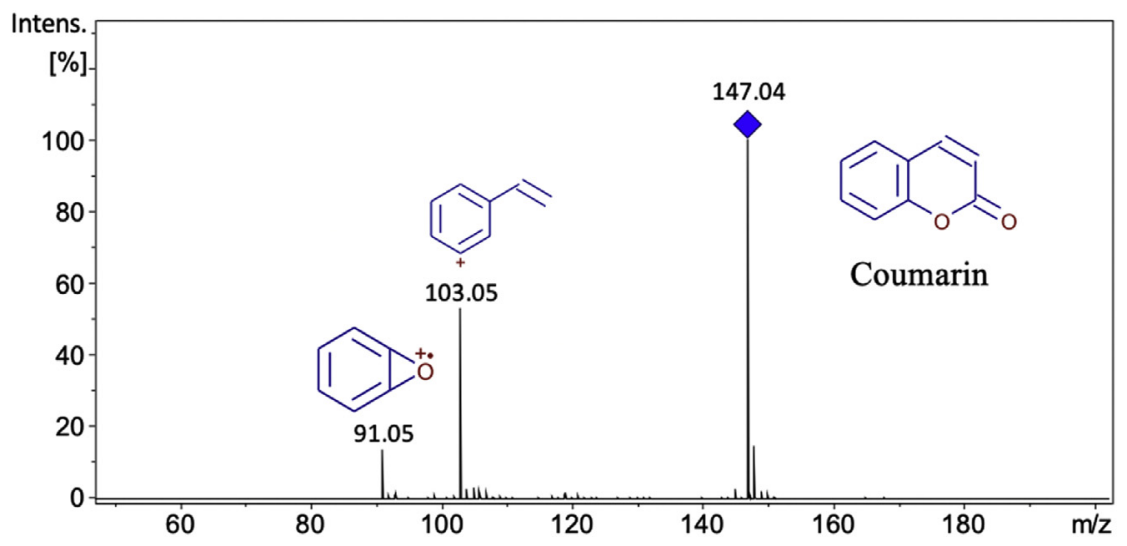

Supplementary Fig. 1. MS/MS spectrum of the marker peak at $\mathrm{m} / \mathrm{z}$ 147.04. This peak was identified as coumarin and the elucidated fragment structures were obtained by Mass Frontier 8.0.
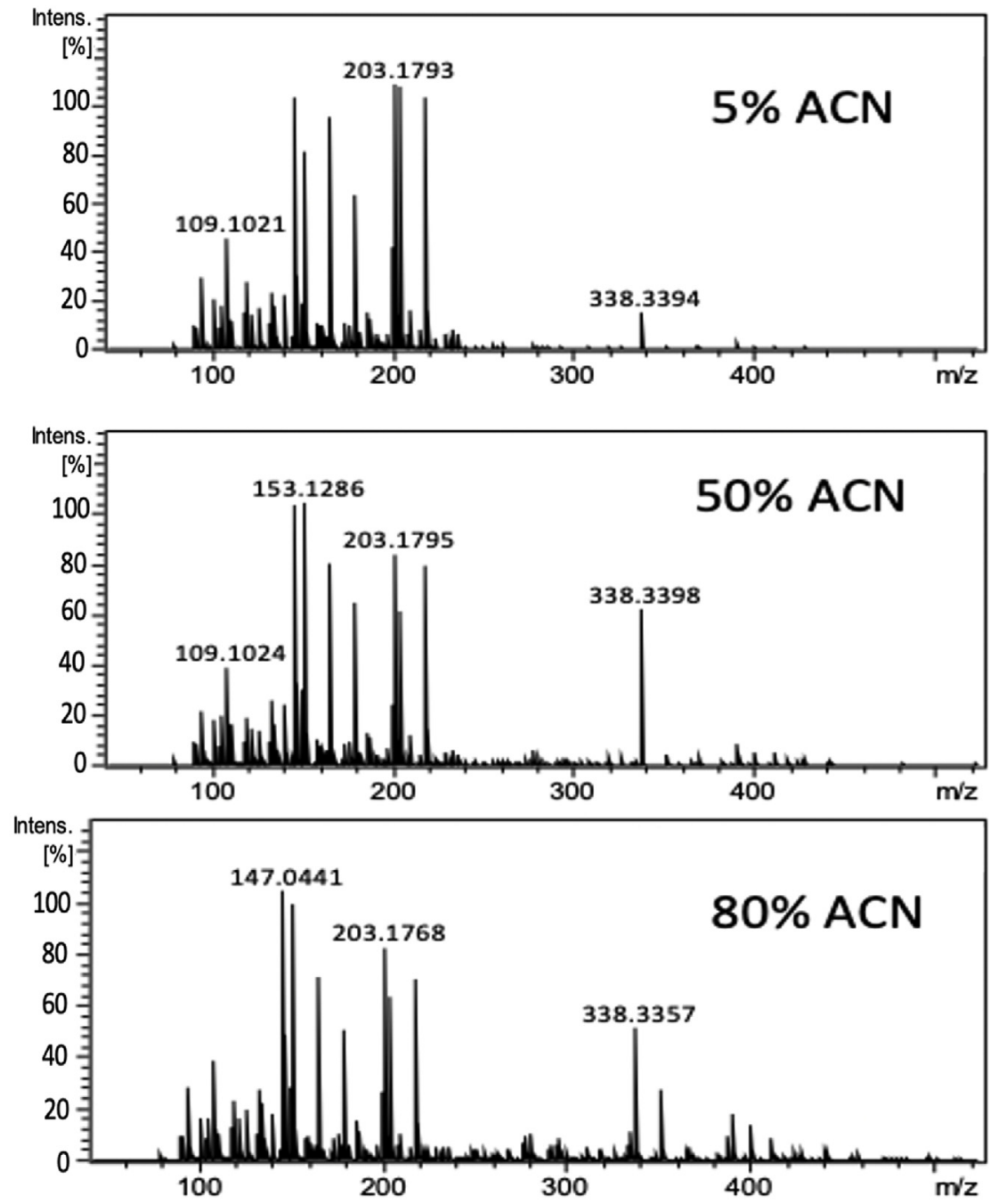

Supplementary Fig. 2. EnESI-MS analysis of Schisandra chinensis using 5\% ACN, 50\% ACN and 80\% ACN as the spray solution. 


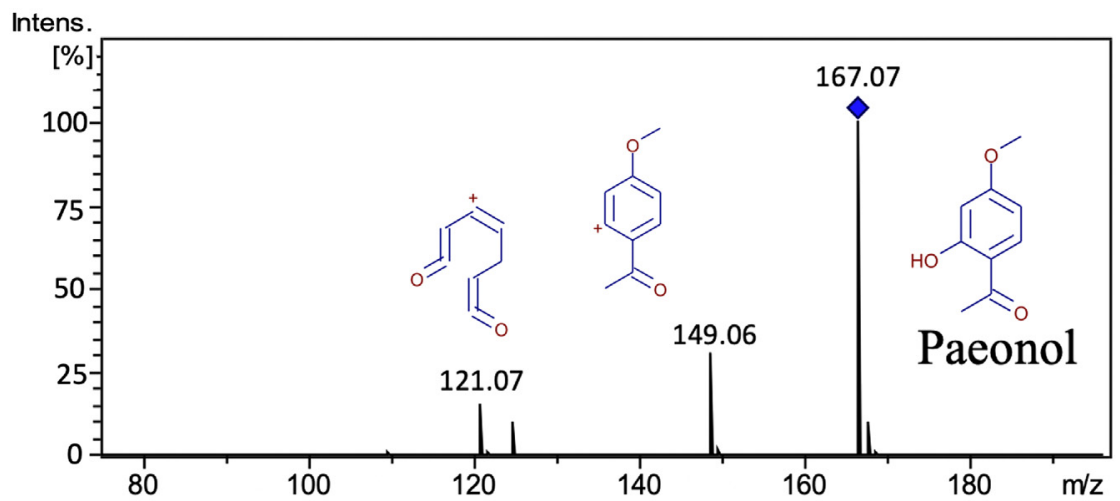

Supplementary Fig. 3. MS/MS spectrum of the marker peak at $\mathrm{m} / \mathrm{z}$ 167.07. This peak was identified as paeonol and the elucidated fragment structures were obtained by Mass Frontier 8.0.

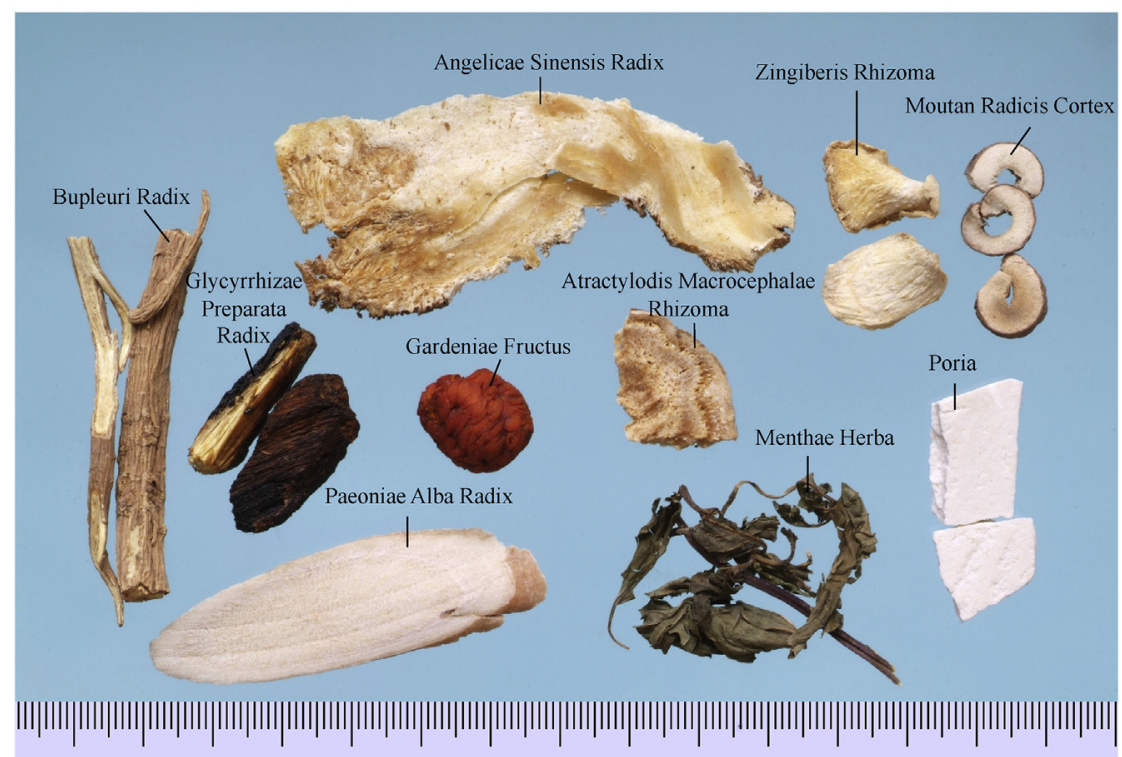

Supplementary Fig. 4. Morphologies of ten herbs in Jia Wei Xiao Yao San. 
(a)

(c)
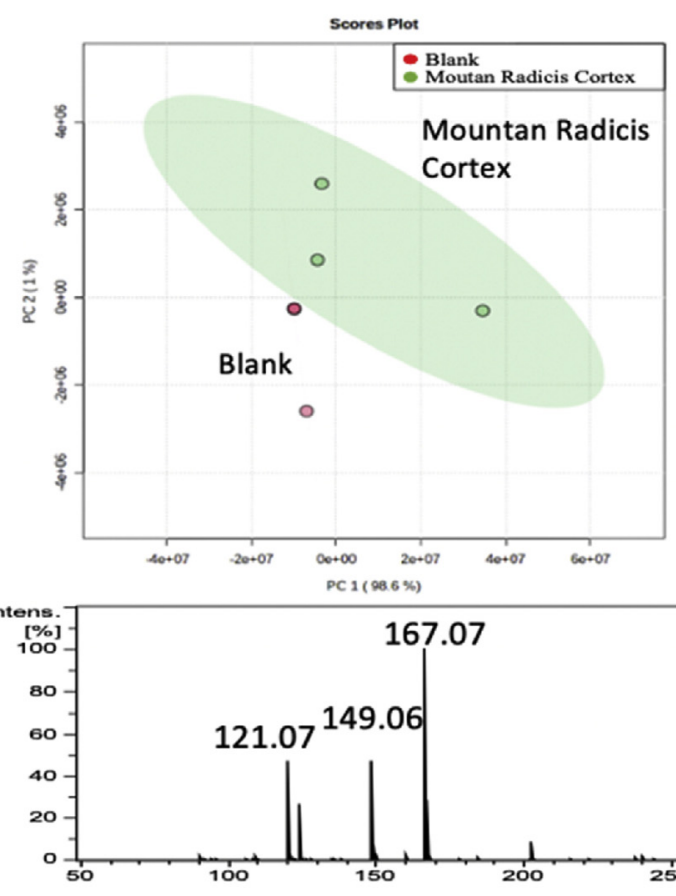

(b)

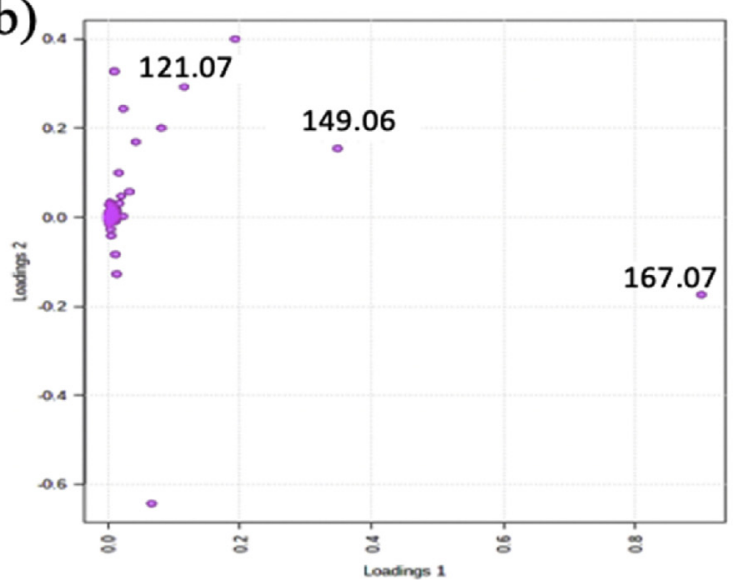

(a)

Store A

Store B

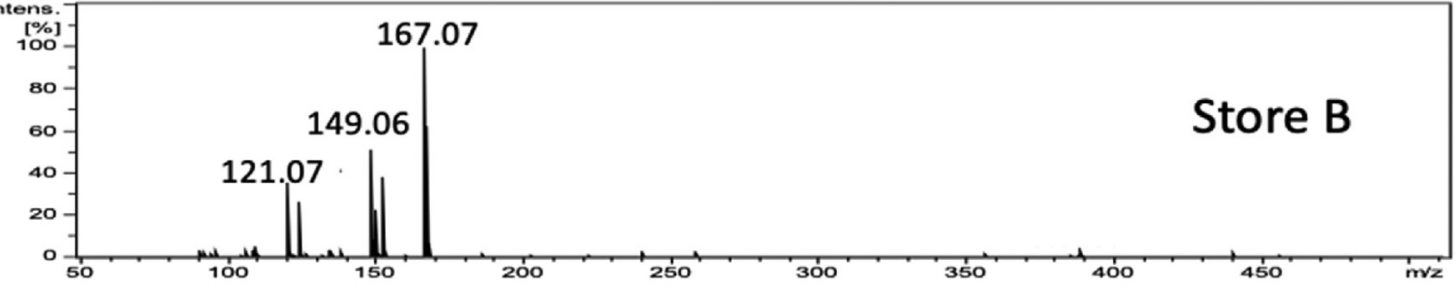

(d)
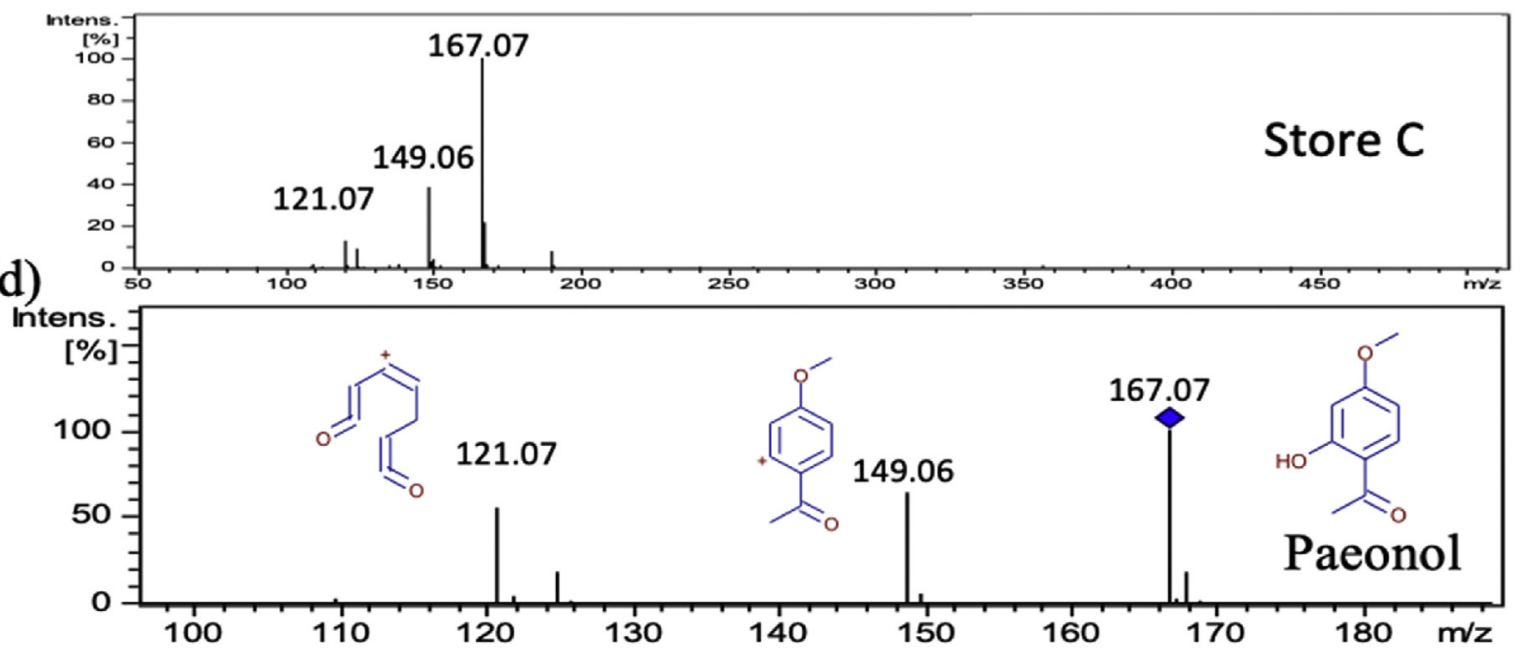

Supplementary Fig. 5. (a) PCA result for Mountan Radicis Cortex $(n=3)$. (b) Loading plot of PC1 and PC2 components. (c) EnESI-MS spectra of Mountan Radicis Cortex from three different stores. (d) MS/MS spectrum of the marker peak at $\mathrm{m} / \mathrm{z} 167.07$. This peak was identified as paeonol, and the elucidated fragment structures were obtained by Mass Frontier 8.0. 

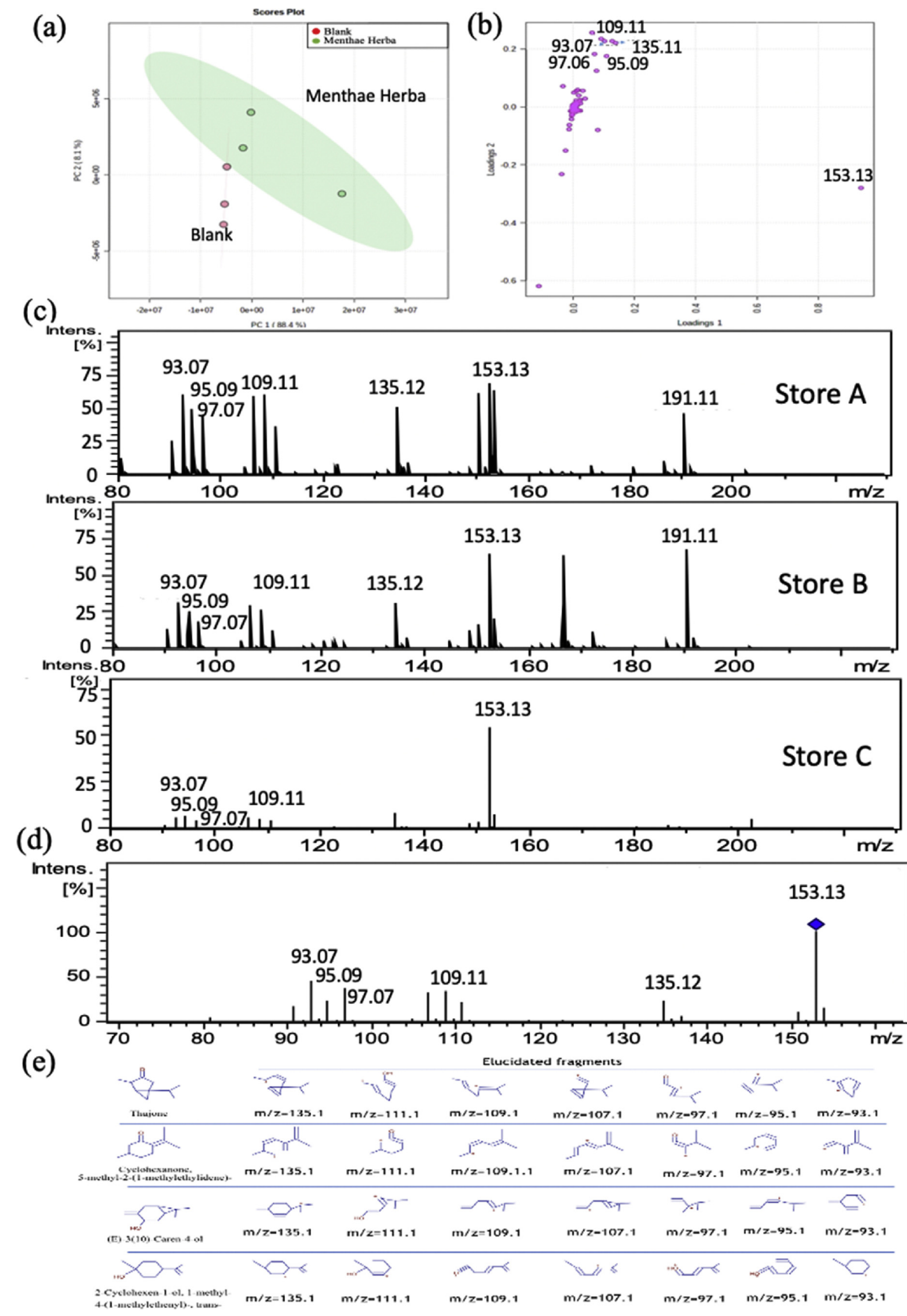

Supplementary Fig. 6. (a) PCA result for Menthae Herba $(n=3)$. (b) Loading plot of PC1 and PC2 components. (c) EnESI-MS spectra of Menthae Herba from three different stores. (d) MS/MS spectrum of the marker peak at $\mathrm{m} / \mathrm{z} 153.13$. (e)The possible identified structures and their fragmented ions deduced by Mass Frontier 8.0. 
(a)

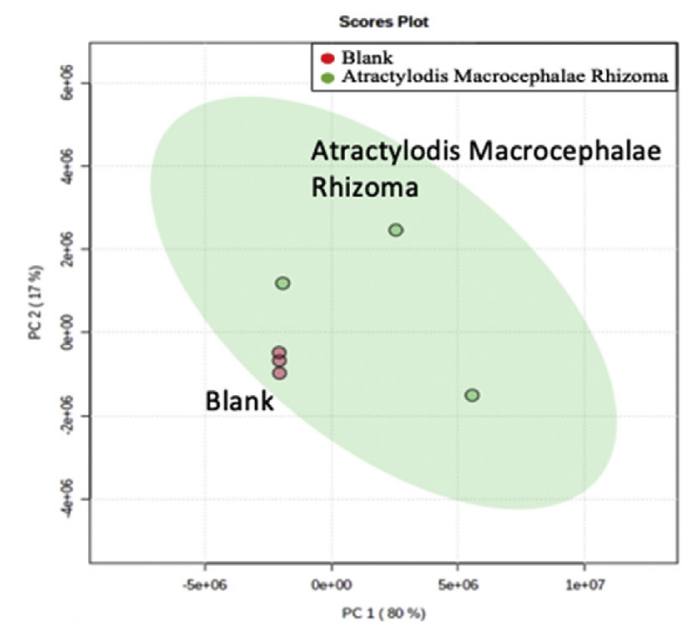

(c)
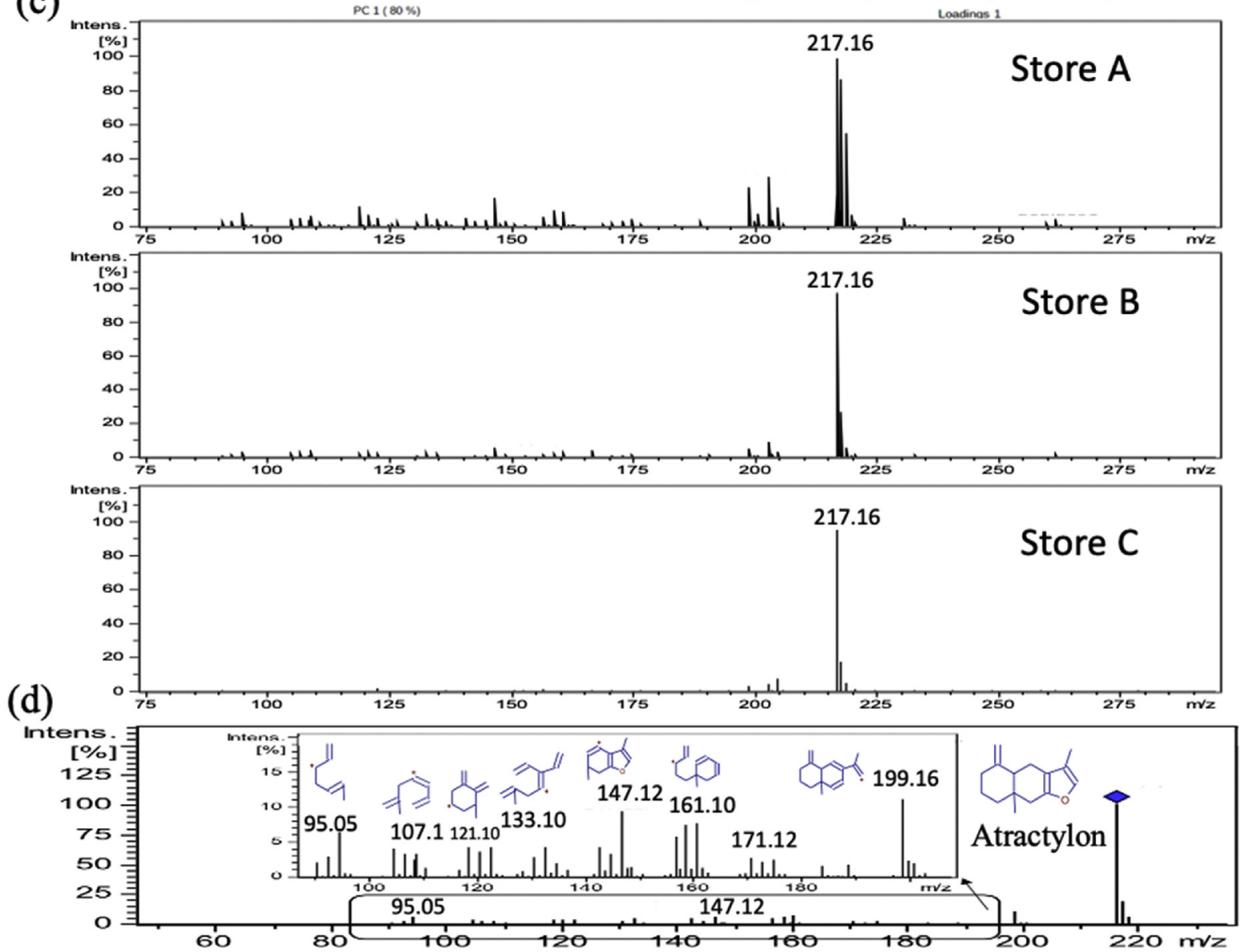

Supplementary Fig. 7. (a) PCA result for Atractylodis Macrocephalae Rhizoma( $=3$ ). (b) Loading plot of PC1 and PC2 components. (c) EnESI-MS spectra of Atractylodis Macrocephalae Rhizoma from three different stores. (d) MS/MS spectrum of the marker peak at m/z 217.16. This peak was identified as atractylon, and the elucidated fragment structures were obtained by Mass Frontier 8.0. 
(a)

(c)

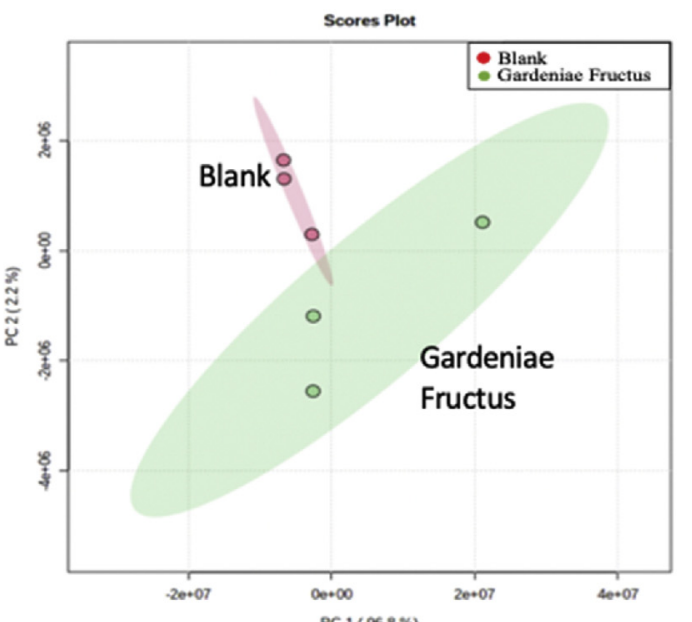

(b)

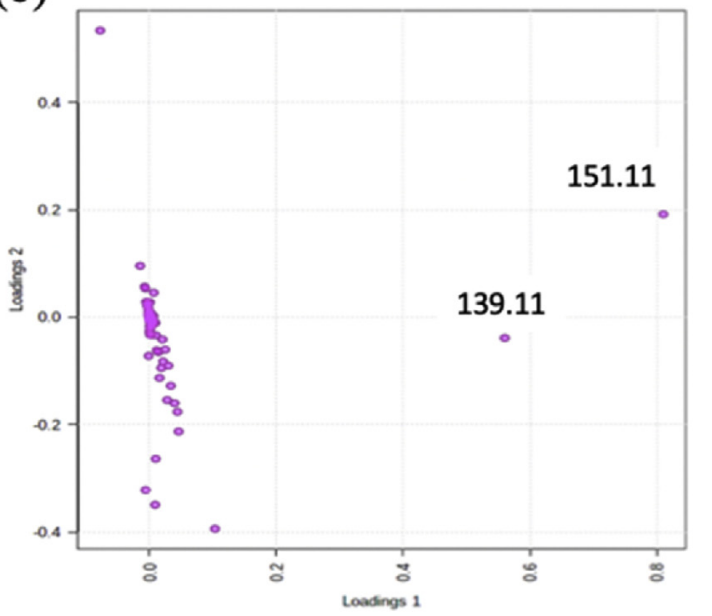

Store A
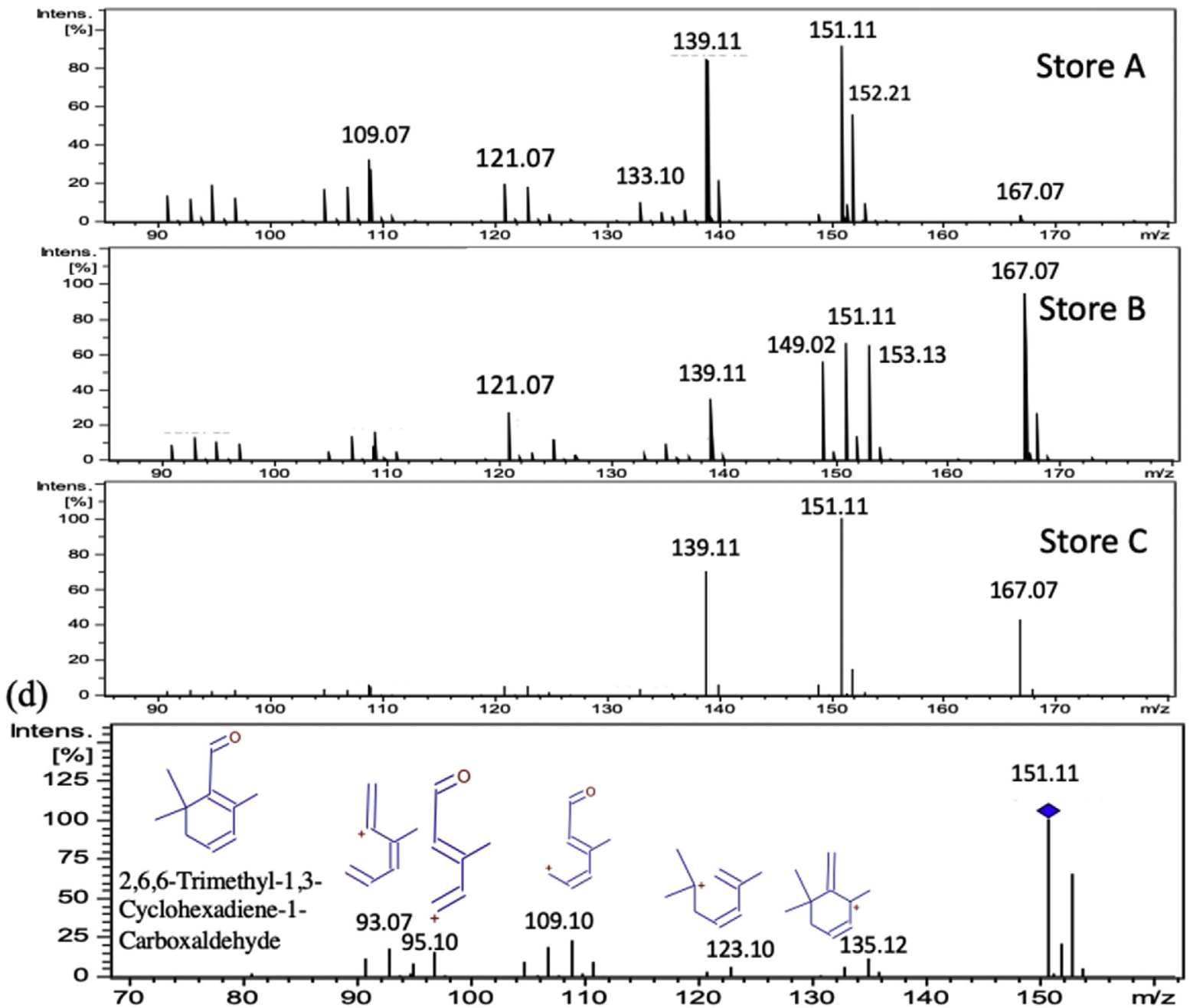

Supplementary Fig. 8. (a) PCA result for Gardeniae Fructus $(n=3)$. (b) Loading plot of PC1 and PC2 components. (c) EnESI-MS spectra of Gardeniae Fructus from three different stores. (d) MS/MS spectrum of the marker peak at $\mathrm{m} / \mathrm{z}$ 151.11. This peak was identified as 2, 6, 6-trimethyl-1, 3cyclohexadiene-1-carboxaldehyde, and the elucidated fragment structures were obtained by Mass Frontier 8.0. 
(a)

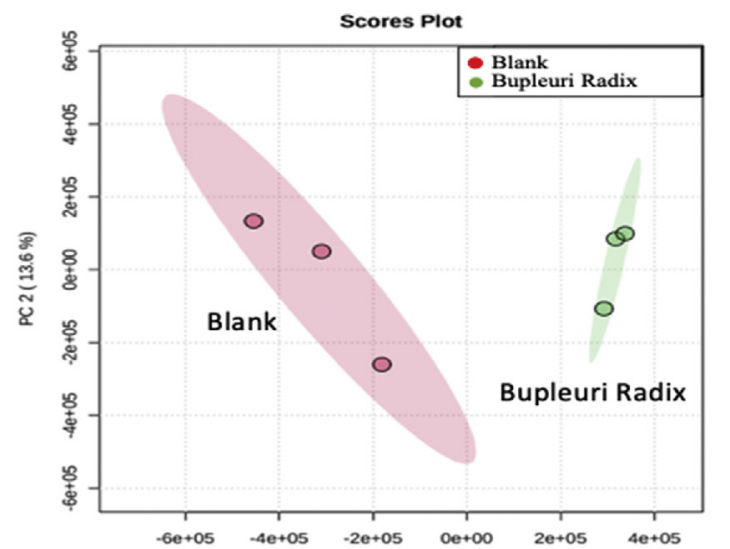

(c)
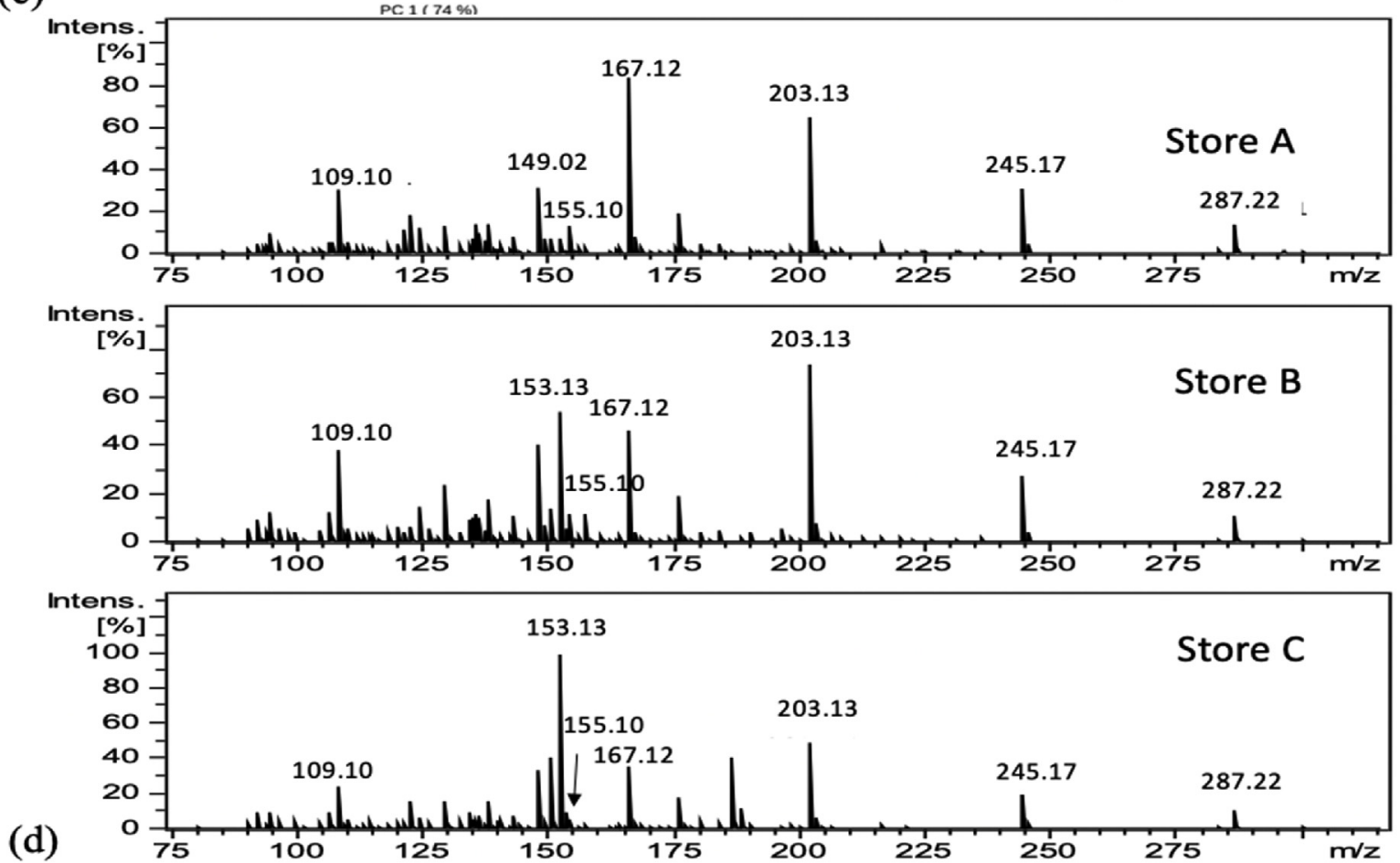

(d)

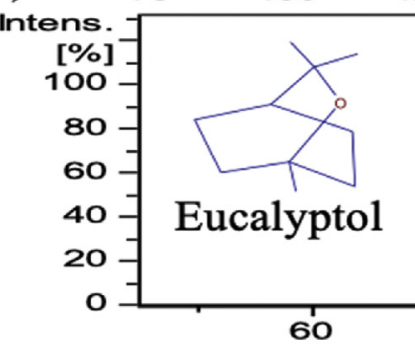

(b)

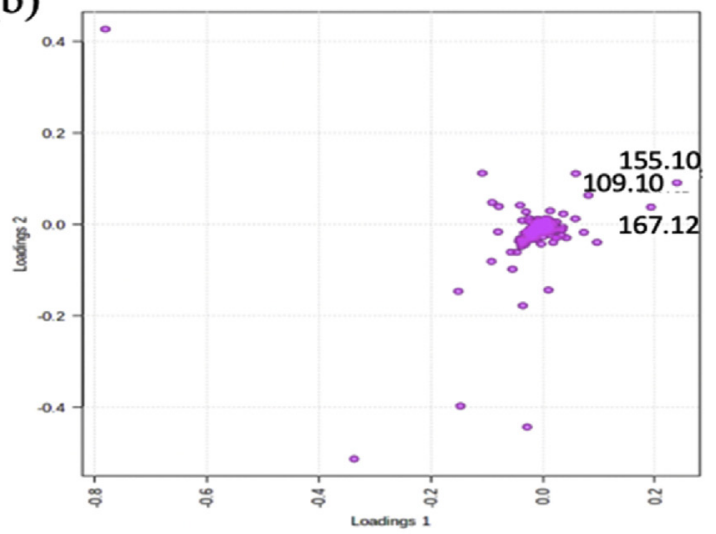

Supplementary Fig. 9. (a) PCA result for Bupleuri Radix $(n=3)$. (b) Loading plot of PC1 and PC2 components. (c) EnESI-MS spectra of Bupleuri Radix from three different stores. (d) MS/MS spectrum of the marker peak at m/z 155.10. This peak was identified as eucalyptol, and the elucidated fragment structures were obtained by Mass Frontier 8.0. 
(a)

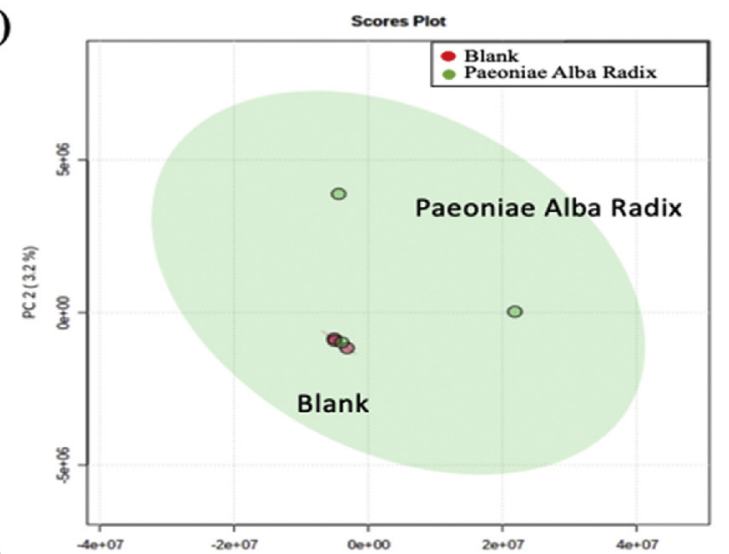

(c)
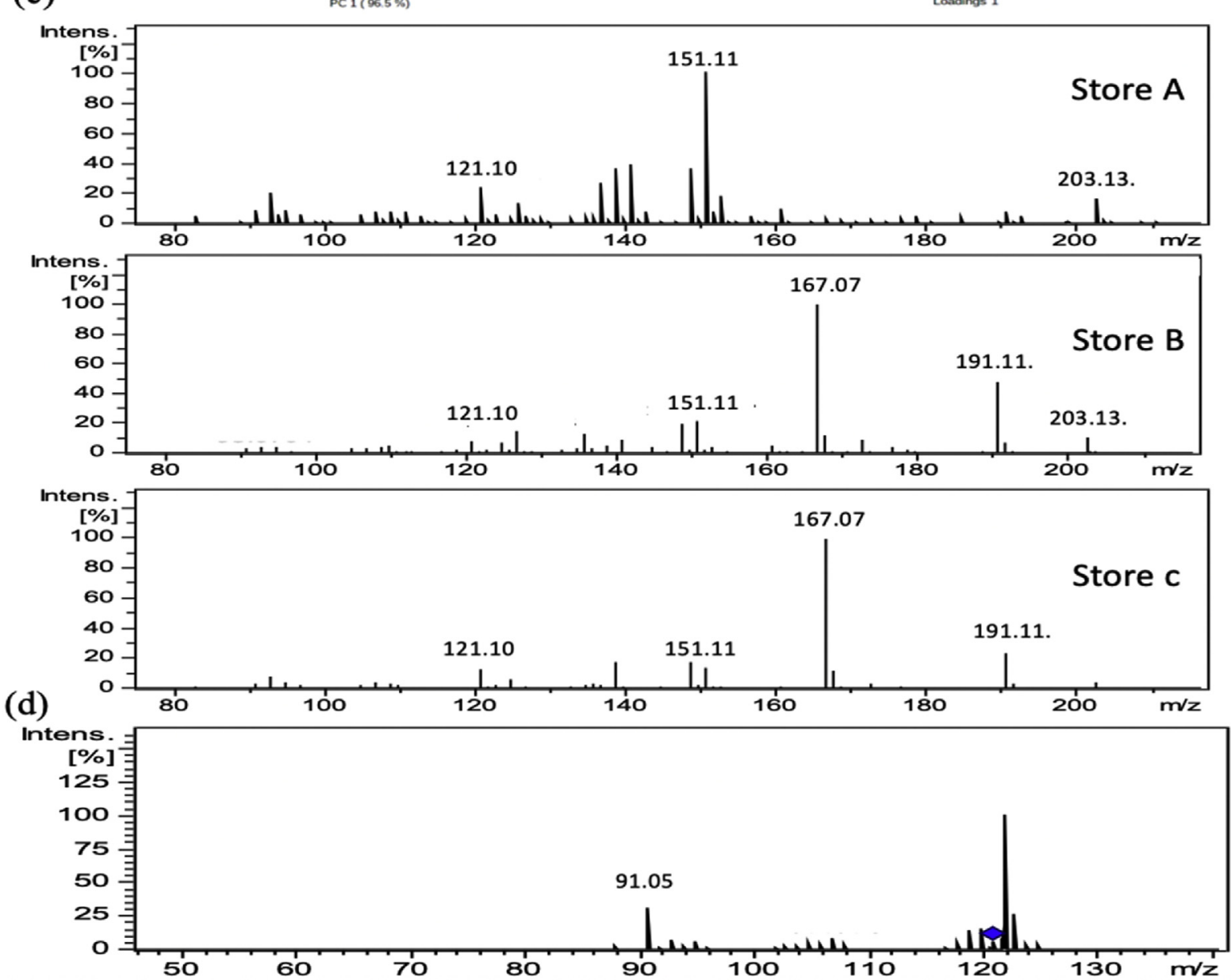

Supplementary Fig. 10. (a) PCA result of Paeoniae Alba Radix $(n=3)$. (b) Loading plot of PC1 and PC2 components. (c) EnESI-MS spectra of Paeoniae Alba Radix from three different stores. (d) MS/MS spectrum of the marker peak at $\mathrm{m} / \mathrm{z} 121.10$.

(b)

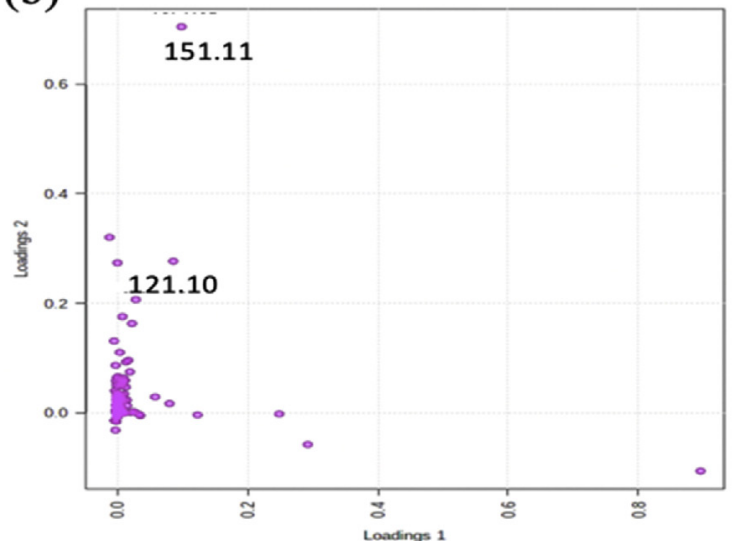

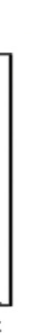


(a)

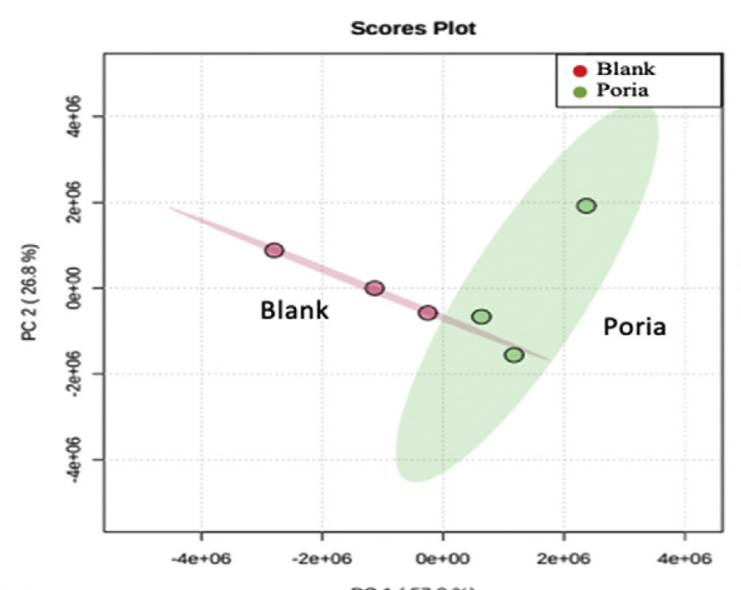

(c)
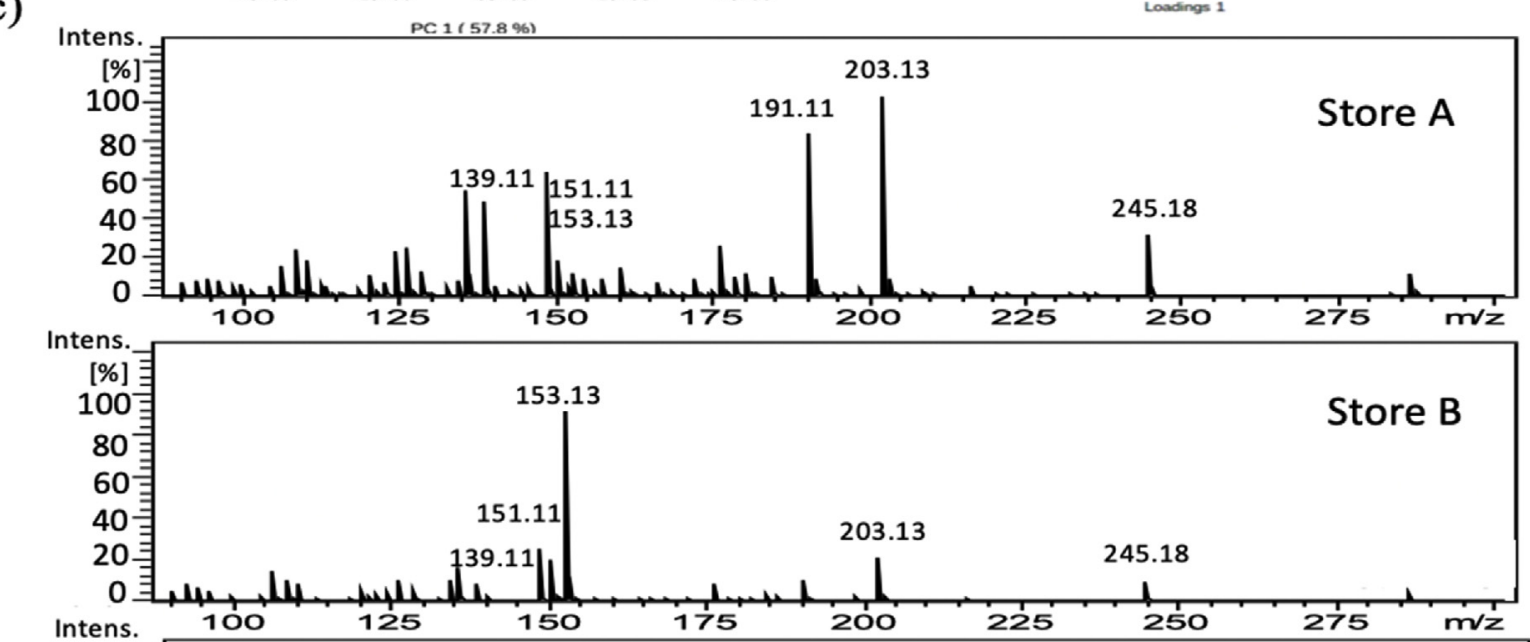

(d)
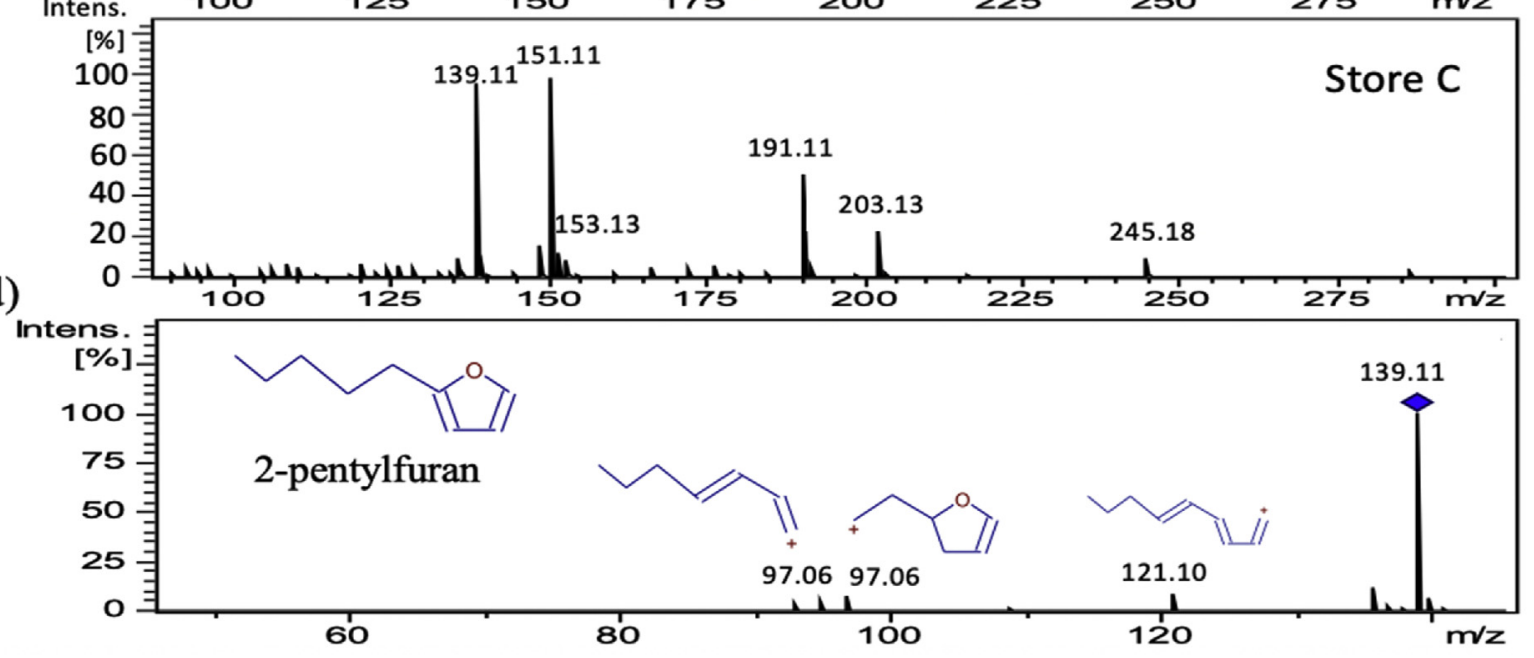

Supplementary Fig. 11. (a) PCA result for Poria $(n=3)$. (b) Loading plot of PC1 and PC2 components. (c) EnESI-MS spectra of Poria from three different stores. (d) MS/MS spectrum of the marker peak at $\mathrm{m} / \mathrm{z}$ 139.11. This peak was identified as 2-pentylfuran, and the elucidated fragment structures were obtained by Mass Frontier 8.0. 
(a)

(c)

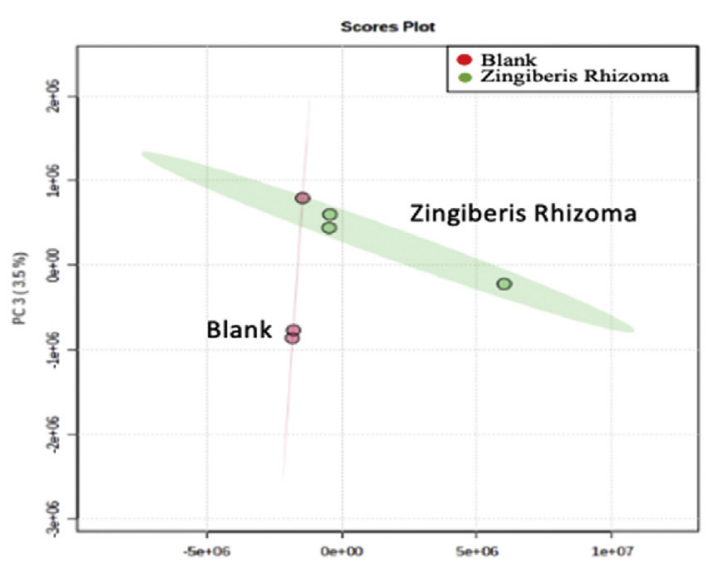

(b)
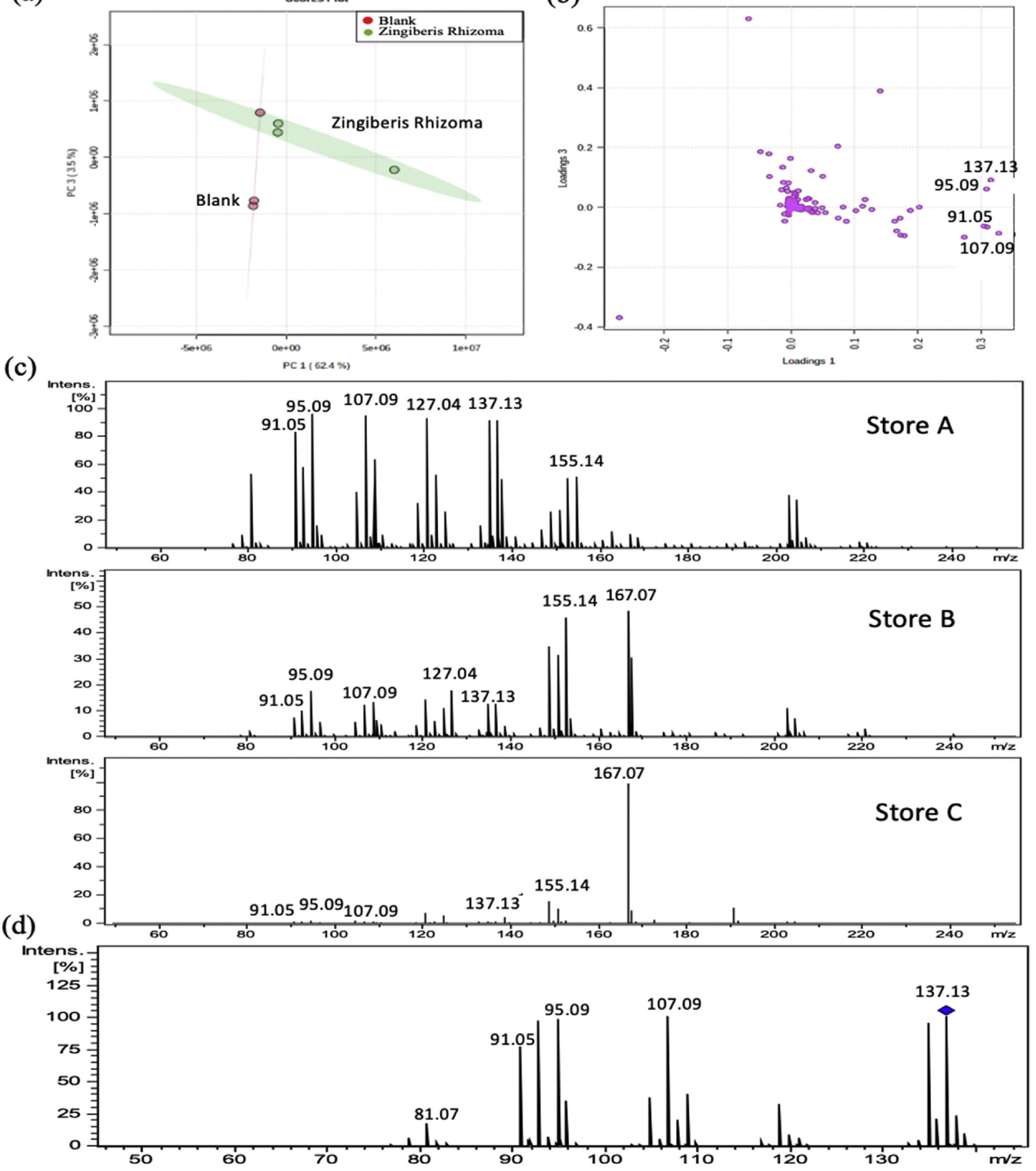

Supplementary Fig. 12. (a) PCA result for Zingiberis Rhizoma $(n=3$ ). (b) Loading plot of PC1 and PC2 components. (c) EnESI-MS spectra of Zingiberis Rhizoma from three different stores. (d) MS/MS spectrum of the marker peak at m/z 137.13. 
(a)

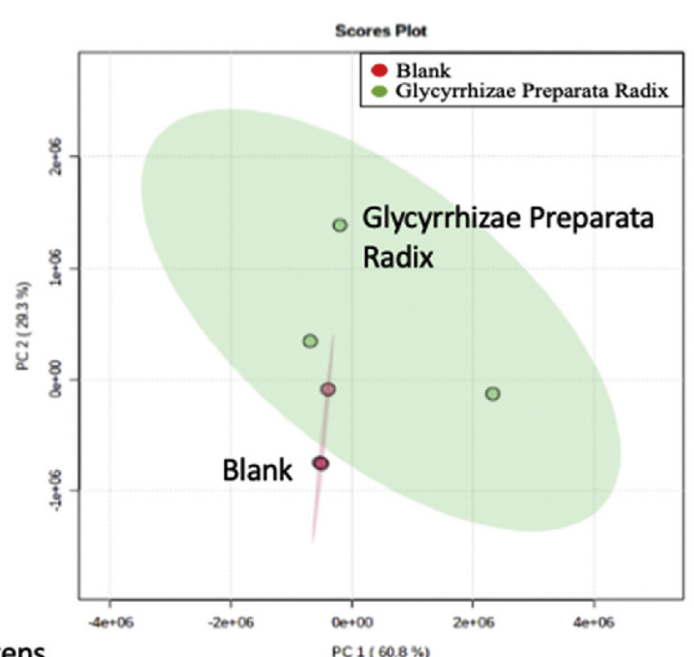

(c) Intens.

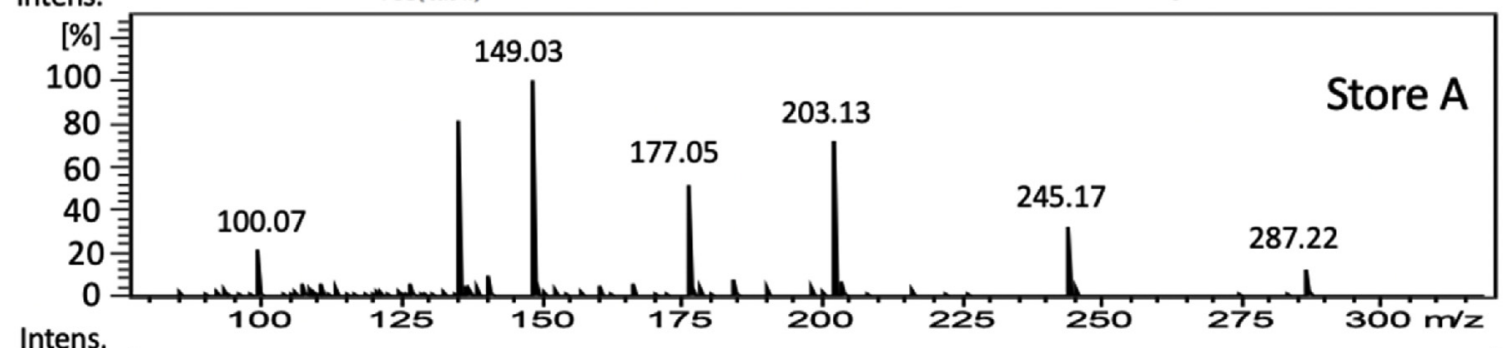

(d) Intens.

(b)
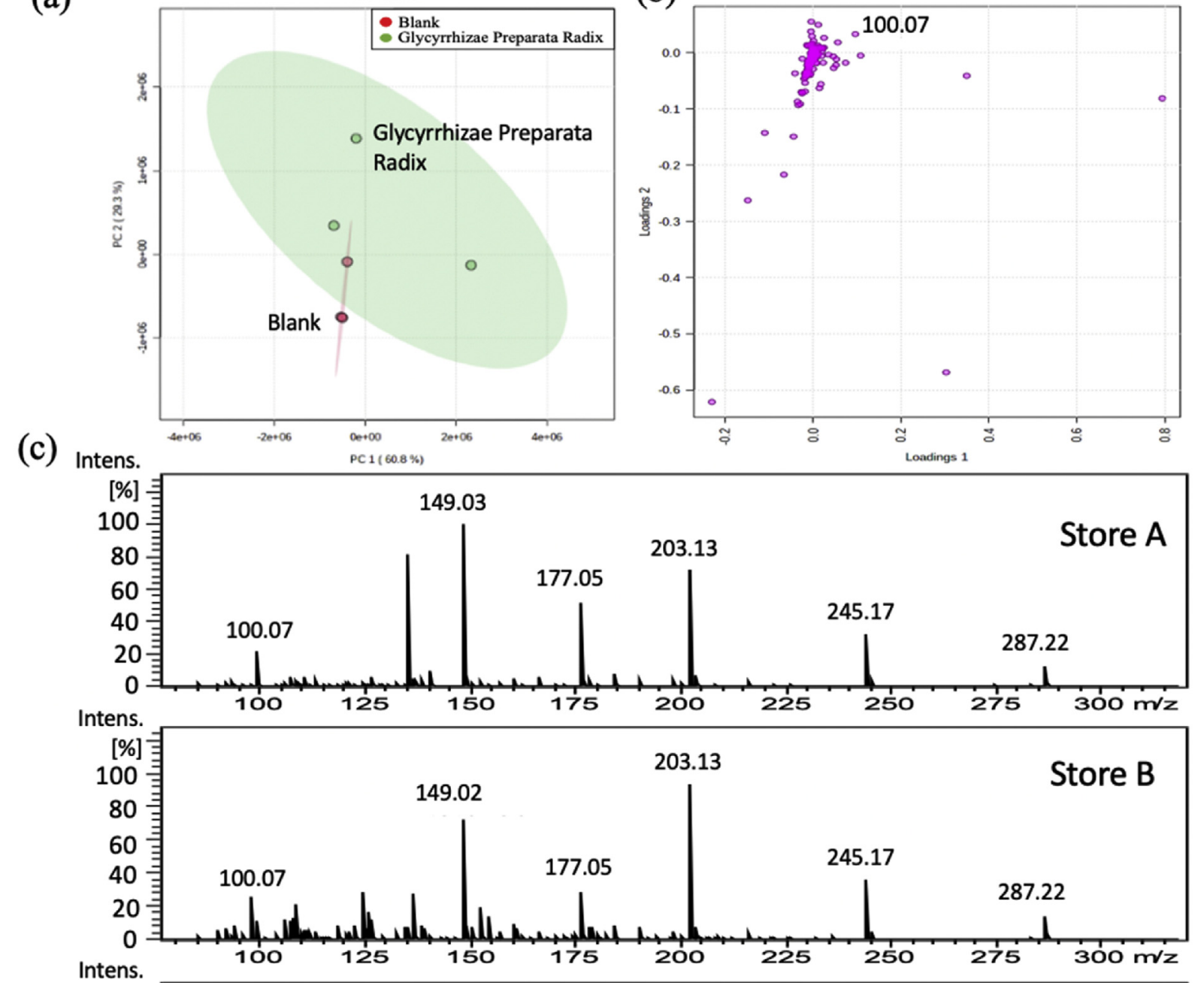

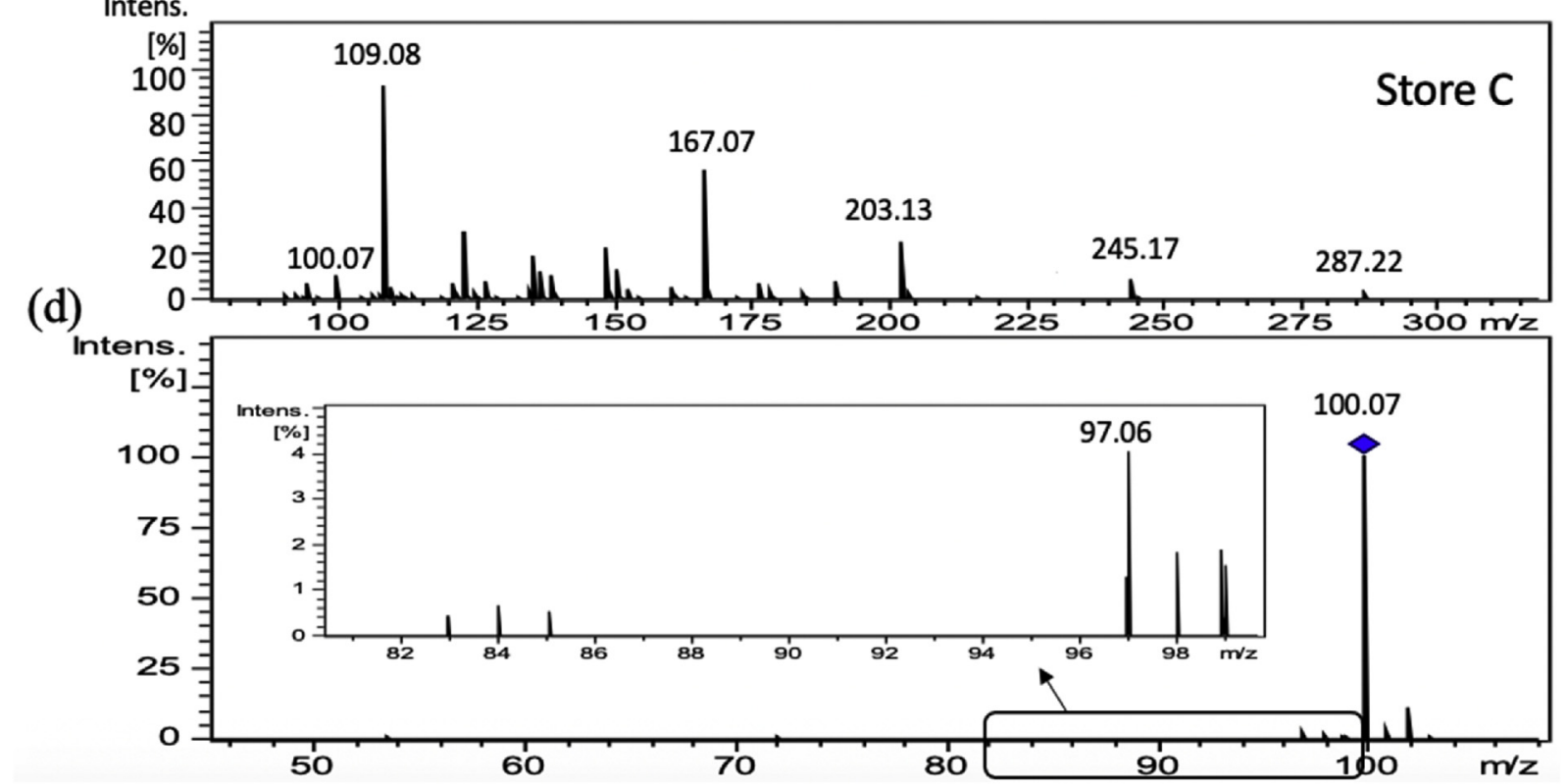

Supplementary Fig. 13. (a) PCA result for Glycyrrhizae Preparata Radix $(n=3)$. (b) Loading plot of PC1 and PC2 components. (c) EnESI-MS spectra of Glycyrrhizae Preparata Radix from three different stores. (d) MS/MS spectrum of the marker peak at m/z 100.07. 


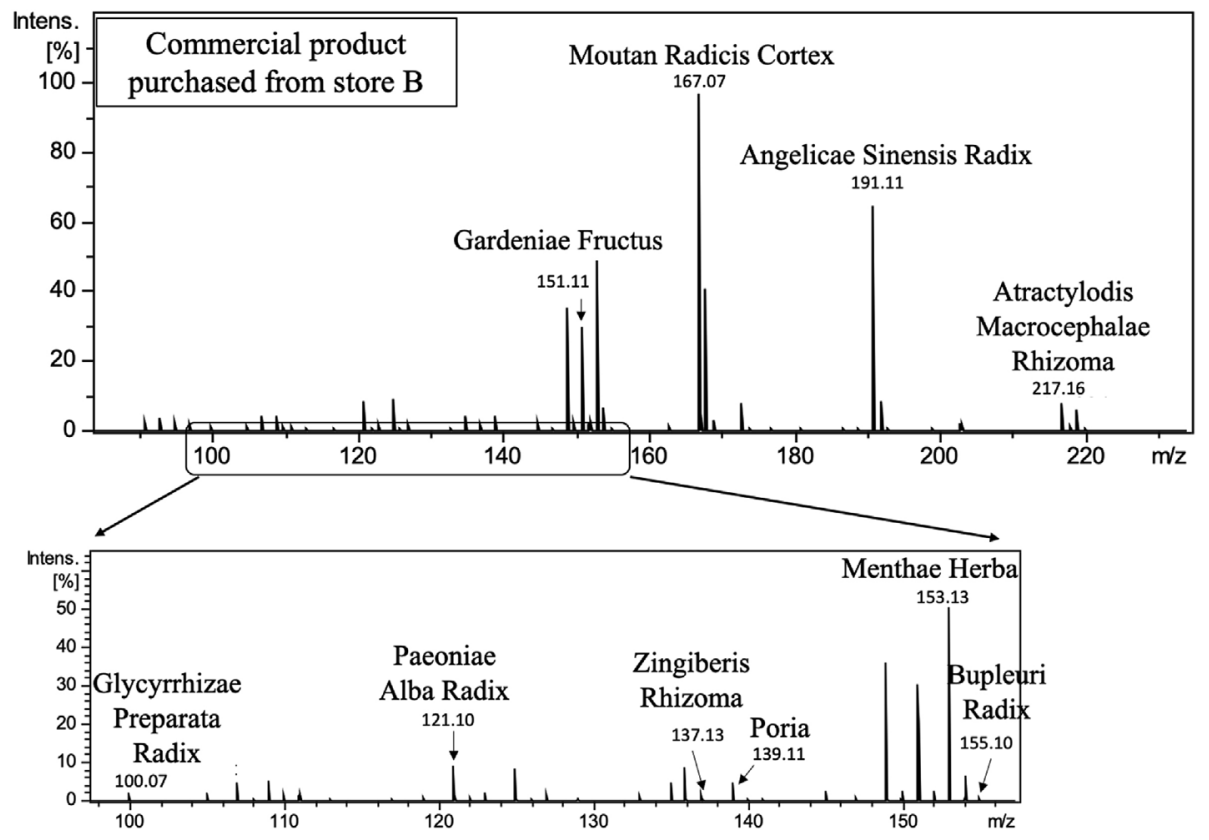

Supplementary Fig. 14. EnESI-MS spectrum of commercial concentrated Jia Wei Xiao Yao San from store B (Sun-Ten Pharmaceutical Co., Taichung, Taiwan).

\section{References}

[1] Zhang WJ, Huai Y, Miao ZP, Qian AR, Wang YH. Systems pharmacology for investigation of the mechanisms of action of traditional Chinese medicine in drug discovery. Front Pharmacol 2019;10 [In English].

[2] Lin CC, Chen KB, Tsai CH, Tsai FJ, Huang $\mathrm{CY}$, Tang $\mathrm{CH}$, et al. Casticin inhibits human prostate cancer DU 145 cell migration and invasion via Ras/Akt/NF-kappaB signaling pathways. J Food Biochem 2019;43:e12902.

[3] Lee HP, Chen PC, Wang SW, Fong YC, Tsai CH, Tsai FJ, et al. Plumbagin suppresses endothelial progenitor cellrelated angiogenesis in vitro and in vivo. J Funct Foods 2019; 52:537-44 [In English].

[4] Huang TY, Peng SF, Huang YP, Tsai CH, Tsai FJ, Huang CY, et al. Combinational treatment of all-trans retinoic acid (ATRA) and bisdemethoxycurcumin (BDMC)-induced apoptosis in liver cancer Hep3B cells. J Food Biochem 2020; 44:e13122.

[5] Zhao Z, Hu Y, Liang Z, Yuen JP, Jiang Z, Leung KS. Authentication is fundamental for standardization of Chinese medicines. Planta Med 2006;72:865-74 [In English].

[6] Huang J, Wong KH, Tay SV, How A, Tam JP. Cysteine-rich peptide fingerprinting as a general method for herbal analysis to differentiate radix astragali and radix hedysarum. Front Plant Sci 2019;10:973 [In English].

[7] Long Q, Li Z, Han B, Gholam Hosseini H, Zhou H, Wang S, et al. Discrimination of two cultivars of alpinia officinarum hance using an electronic nose and gas chromatographymass spectrometry coupled with chemometrics. Sensors (Basel) 2019;19.

[8] Zhou H, Luo D, GholamHosseini H, Li Z, He J. Identification of Chinese herbal medicines with electronic nose technology: applications and challenges. Sensors (Basel) 2017;17.

[9] Chen H, Venter A, Cooks RG. Extractive electrospray ionization for direct analysis of undiluted urine, milk and other complex mixtures without sample preparation. Chem Commun (Camb) 2006:2042-4 [In English].
[10] Law WS, Wang R, Hu B, Berchtold C, Meier L, Chen H, et al. On the mechanism of extractive electrospray ionization. Anal Chem 2010;82:4494-500.

[11] Chen H, Sun Y, Wortmann A, Gu H, Zenobi R. Differentiation of maturity and quality of fruit using noninvasive extractive electrospray ionization quadrupole time-of-flight mass spectrometry. Anal Chem 2007;79:1447-55 [In English].

[12] Ke M, Zhang H, Ding J, Xiong X, Li F, Chingin K, et al. Generating supercharged protein ions for breath analysis by extractive electrospray ionization mass spectrometry. Anal Chem 2019;91:3215-20.

[13] Zhu L, Gamez G, Chen HW, Huang HX, Chingin K, Zenobi R. Real-time, on-line monitoring of organic chemical reactions using extractive electrospray ionization tandem mass spectrometry. Rapid Commun Mass Spectrom 2008;22: 2993-8.

[14] Wang S, Li F, Liu Y, Zhao H, Chen H. High-throughput screening of toxic substances by extractive electrospray ionization mass spectrometry and their identification via databank construction. Anal Bioanal Chem 2019;411: 4049-54

[15] Taiwan herbal Pharmacopeia. 3rd ed. English version: Ministry Health and Welfare, Taiwan, R.O.C.; 2019.

[16] Chiang MH, Chang LW, Wang JW, Lin LC, Tsai TH. Herbdrug pharmacokinetic interaction of a traditional Chinese medicine jia-wei-xiao-yao-san with 5-Fluorouracil in the blood and brain of rat using microdialysis. Evid Based Complement Alternat Med 2015;2015:729679.

[17] Ren Z, Nie B, Liu T, Yuan F, Feng F, Zhang Y, et al. Simultaneous determination of coumarin and its derivatives in tobacco products by liquid chromatography-tandem mass spectrometry. Molecules 2016;21:1511-23.

[18] Kwon MH, Jeong JS, Ryu J, Cho YW, Kang HE. Simultaneous determination of saikosaponin a, paeonol, and imperatorin, components of DA-9805, in rat plasma by LC-MS/MS and application to a pharmacokinetic study. J Chromatogr B Analyt Technol Biomed Life Sci 2017;1068-1069:289-96. 
[19] Diao X, Ma Z, Wang H, Zhong D, Zhang Y, Jin J, et al. Simultaneous quantitation of 3-n-butylphthalide (NBP) and its four major metabolites in human plasma by LC-MS/MS using deuterated internal standards. J Pharm Biomed Anal 2013;78-79:19-26.

[20] Yi L, Liang Y, Wu H, Yuan D. The analysis of radix Angelicae Sinensis (Danggui). J Chromatogr A 2009;1216: 1991-2001.

[21] Hu S, Shen G, Zhao W, Wang F, Jiang X, Huang D. Paeonol, the main active principles of Paeonia moutan, ameliorates alcoholic steatohepatitis in mice. J Ethnopharmacol 2010;128: 100-6 [In English].
[22] Thurman EM, Ferrer I, Pozo OJ, Sancho JV, Hernandez F. The even-electron rule in electrospray mass spectra of pesticides. Rapid Commun Mass Spectrom 2007;21:3855-68.

[23] Xing J, Sun HM, Li ZY, Qin XM. Comparison of volatile components between raw and vinegar baked radix Bupleuri by GC-MS based metabolic fingerprinting approach. Evid Based Complement Alternat Med 2015;2015:653791.

[24] Cao G, Shan Q, Li X, Cong X, Zhang Y, Cai H, et al. Analysis of fresh Mentha haplocalyx volatile components by comprehensive two-dimensional gas chromatography and high-resolution time-of-flight mass spectrometry. Analyst 2011;136:4653-61. 